\title{
Construction of pathological maximally monotone operators on non-reflexive Banach spaces
}

\author{
Heinz H. Bauschke, Jonathan M. Borwein† Xianfu Wang
}

August 6, 2011

\begin{abstract}
In this paper, we construct maximally monotone operators that are not of Gossez's dense-type (D) in many nonreflexive spaces. Many of these operators also fail to possess the Brønsted-Rockafellar (BR) property. Using these operators, we show that the partial inf-convolution of two $\mathrm{BC}$-functions will not always be a $\mathrm{BC}$-function. This provides a negative answer to a challenging question posed by Stephen Simons. Among other consequences, we deduce that every Banach space which contains an isomorphic copy of the James space $\mathbf{J}$ or its dual $\mathbf{J}^{*}$, or $c_{0}$ or its dual $\ell^{1}$, admits a non type (D) operator.
\end{abstract}

\section{Mathematics Subject Classification:}

Primary 47A06, 47H05; Secondary 47B65, 47N10, 90C25

Keywords: Adjoint, BC-function, Fitzpatrick function, James space, linear relation, maximally monotone operator, monotone operator, multifunction, operator of type (BR), operator of type (D), operator of type (NI), partial inf-convolution, Schauder basis, set-valued operator, skew operator, space of type (D), uniqueness of extensions, subdifferential operator.

*Mathematics, Irving K. Barber School, University of British Columbia, Kelowna, B.C. V1V 1V7, Canada. E-mail: heinz.bauschke@ubc.ca.

$\dagger$ CARMA, University of Newcastle, Newcastle, New South Wales 2308, Australia. E-mail: jonathan. borwein@newcastle.edu. au. Distinguished Professor King Abdulaziz University, Jeddah.

${ }_{\ddagger}^{\ddagger}$ Mathematics, Irving K. Barber School, University of British Columbia, Kelowna, B.C. V1V 1V7, Canada. E-mail: shawn.wang@ubc.ca.

$\S$ Mathematics, Irving K. Barber School, University of British Columbia, Kelowna, B.C. V1V 1V7, Canada. E-mail: 1jinyao@interchange.ubc.ca. 


\section{Preliminaries}

Throughout this paper, we assume that $X$ is a real Banach space with norm $\|\cdot\|$, that $X^{*}$ is the continuous dual of $X$, and that $X$ and $X^{*}$ are paired by $\langle\cdot, \cdot\rangle$. As usual, we identify $X$ with its canonical image in the bidual space $X^{* *}$. Furthermore, $X \times X^{*}$ and $\left(X \times X^{*}\right)^{*}:=X^{*} \times X^{* *}$ are likewise paired via $\left\langle\left(x, x^{*}\right),\left(y^{*}, y^{* *}\right)\right\rangle:=\left\langle x, y^{*}\right\rangle+\left\langle x^{*}, y^{* *}\right\rangle$, where $\left(x, x^{*}\right) \in X \times X^{*}$ and $\left(y^{*}, y^{* *}\right) \in X^{*} \times X^{* *}$.

Let $A$ : $X \rightrightarrows X^{*}$ be a set-valued operator (also known as a multifunction) from $X$ to $X^{*}$, i.e., for every $x \in X, A x \subseteq X^{*}$, and let gra $A:=\left\{\left(x, x^{*}\right) \in X \times X^{*} \mid x^{*} \in A x\right\}$ be the graph of $A$. The domain of $A$ is $\operatorname{dom} A:=\{x \in X \mid A x \neq \varnothing\}$, and $\operatorname{ran} A:=A(X)$ for the range of $A$. Recall that $A$ is monotone if

$$
\left\langle x-y, x^{*}-y^{*}\right\rangle \geq 0, \quad \forall\left(x, x^{*}\right) \in \operatorname{gra} A \forall\left(y, y^{*}\right) \in \operatorname{gra} A,
$$

and maximally monotone if $A$ is monotone and $A$ has no proper monotone extension (in the sense of graph inclusion). Let $A: X \rightrightarrows X^{*}$ be monotone and $\left(x, x^{*}\right) \in X \times X^{*}$. We say $\left(x, x^{*}\right)$ is monotonically related to gra $A$ if

$$
\left\langle x-y, x^{*}-y^{*}\right\rangle \geq 0, \quad \forall\left(y, y^{*}\right) \in \operatorname{gra} A .
$$

We now recall the three fundamental subclasses of maximally monotone operators.

Definition 1.1 Let $A: X \rightrightarrows X^{*}$ be maximally monotone. Then three key types of monotone operators are defined as follows.

(i) $A$ is of dense type or type (D) (1971, [19] and [28]) if for every $\left(x^{* *}, x^{*}\right) \in X^{* *} \times X^{*}$ with

$$
\inf _{\left(a, a^{*}\right) \in \operatorname{gra} A}\left\langle a-x^{* *}, a^{*}-x^{*}\right\rangle \geq 0,
$$

there exist a bounded net $\left(a_{\alpha}, a_{\alpha}^{*}\right)_{\alpha \in \Gamma}$ in gra $A$ such that $\left(a_{\alpha}, a_{\alpha}^{*}\right)_{\alpha \in \Gamma}$ weak ${ }^{*} \times$ strong converges to $\left(x^{* *}, x^{*}\right)$.

(ii) $A$ is of type negative infimum (NI) (1996, [32]) if

$$
\sup _{\left(a, a^{*}\right) \in \operatorname{gra} A}\left(\left\langle a, x^{*}\right\rangle+\left\langle a^{*}, x^{* *}\right\rangle-\left\langle a, a^{*}\right\rangle\right) \geq\left\langle x^{* *}, x^{*}\right\rangle, \quad \forall\left(x^{* *}, x^{*}\right) \in X^{* *} \times X^{*} .
$$

(iii) $A$ is of "Brønsted-Rockafellar" (BR) type (1999, [37]) if whenever $\left(x, x^{*}\right) \in X \times X^{*}$, $\alpha, \beta>0$ while

$$
\inf _{\left(a, a^{*}\right) \in \operatorname{gra} A}\left\langle x-a, x^{*}-a^{*}\right\rangle>-\alpha \beta
$$

then there exists $\left(b, b^{*}\right) \in \operatorname{gra} A$ such that $\|x-b\|<\alpha,\left\|x^{*}-b^{*}\right\|<\beta$. 
As we shall see below in Fact 2.7, it is now known that the first two classes coincide. This coincidence is central to many of our proofs. Fact 2.11 also shows us that every maximally monotone operator of type (D) is of type (BR)(The converse fails, see Example 4.1](xiii), ). Moreover, in reflexive space every maximally monotone operator is of type (D), as is the subdifferential operator of every closed convex function on a Banach space. While monotone operator theory is rather complete in reflexive space - and for type (D) operators in general space - the general situation is less clear [11, 9]. Hence our continuing interest in operators which are not of type (D).

We shall say a Banach space $X$ is of type (D) [9] if every maximally monotone operator on $X$ is of type (D). At present the only known type (D) spaces are the reflexive spaces; and our work here suggests that there are no non-reflexive type (D) spaces. In [11, Exercise 9.6.3] such spaces were called (NI) spaces and some potential non-reflexive examples were conjectured; all of which are ruled out by our current work. In [11, Theorem 9.79] a variety of the pleasant properties of type (D) spaces was listed.

\section{$1.1 \quad$ More preliminary technicalities}

Maximal monotone operators have proven to be a potent class of objects in modern Optimization and Analysis; see, e.g., [7, 8, 9], the books [6, 11, 13, 27, 33, 35, 31, 42] and the references therein.

We adopt standard notation used in these books especially [11, Chapter 2] and [7, 33, 35]: Given a subset $C$ of $X$, the indicator function of $C$, written as $\iota_{C}$, is defined at $x \in X$ by

$$
\iota_{C}(x):= \begin{cases}0, & \text { if } x \in C ; \\ +\infty, & \text { otherwise. }\end{cases}
$$

The closed unit ball is $B_{X}:=\{x \in X \mid\|x\| \leq 1\}$, and $\mathbb{N}:=\{1,2,3, \ldots\}$.

Let $\alpha, \beta \in \mathbb{R}$. In the sequel it will also be useful to let $\delta_{\alpha, \beta}$ be defined by $\delta_{\alpha, \beta}:=1$, if $\alpha=\beta ; \delta_{\alpha, \beta}:=0$, otherwise.

For a subset $C^{*}$ of $X^{*},{\overline{C^{*}}}^{\mathrm{w}^{*}}$ is the weak* closure of $C^{*}$. If $Z$ is a real Banach space with dual $Z^{*}$ and a set $S \subseteq Z$, we denote $S^{\perp}$ by $S^{\perp}:=\left\{z^{*} \in Z^{*} \mid\left\langle z^{*}, s\right\rangle=0, \quad \forall s \in S\right\}$. Given a subset $D$ of $Z^{*}$, we define $D_{\perp}$ [29] by $D_{\perp}:=\left\{z \in Z \mid\left\langle z, d^{*}\right\rangle=0, \quad \forall d^{*} \in D\right\}$.

The adjoint of an operator $A$, written $A^{*}$, is defined by

$$
\operatorname{gra} A^{*}:=\left\{\left(x^{* *}, x^{*}\right) \in X^{* *} \times X^{*} \mid\left(x^{*},-x^{* *}\right) \in(\operatorname{gra} A)^{\perp}\right\} .
$$

We say $A$ is a linear relation if gra $A$ is a linear subspace. We say that $A$ is skew if gra $A \subseteq$ $\operatorname{gra}\left(-A^{*}\right)$; equivalently, if $\left\langle x, x^{*}\right\rangle=0, \forall\left(x, x^{*}\right) \in \operatorname{gra} A$. Furthermore, $A$ is symmetric if 
$\operatorname{gra} A \subseteq \operatorname{gra} A^{*}$; equivalently, if $\left\langle x, y^{*}\right\rangle=\left\langle y, x^{*}\right\rangle, \forall\left(x, x^{*}\right),\left(y, y^{*}\right) \in \operatorname{gra} A$. We define the symmetric part and the skew part of $A$ via

$$
P:=\frac{1}{2} A+\frac{1}{2} A^{*} \quad \text { and } \quad S:=\frac{1}{2} A-\frac{1}{2} A^{*},
$$

respectively. It is easy to check that $P$ is symmetric and that $S$ is skew. Let $A: X \rightrightarrows X^{*}$ be monotone and $S$ be a subspace of $X$. We say $A$ is $S$-saturated [35] if

$$
A x+S^{\perp}=A x, \quad \forall x \in \operatorname{dom} A .
$$

We say a maximally monotone operator $A: X \rightrightarrows X^{*}$ is unique if all maximally monotone extensions of $A$ (in the sense of graph inclusion) in $X^{* *} \times X^{*}$ coincide.

Let $f: X \rightarrow]-\infty,+\infty]$. Then $\operatorname{dom} f:=f^{-1}(\mathbb{R})$ is the domain of $f$, and $f^{*}: X^{*} \rightarrow$ $[-\infty,+\infty]: x^{*} \mapsto \sup _{x \in X}\left(\left\langle x, x^{*}\right\rangle-f(x)\right)$ is the Fenchel conjugate of $f$. We say $f$ is proper if $\operatorname{dom} f \neq \varnothing$. Let $f$ be proper. The subdifferential of $f$ is defined by

$$
\partial f: X \rightrightarrows X^{*}: x \mapsto\left\{x^{*} \in X^{*} \mid(\forall y \in X)\left\langle y-x, x^{*}\right\rangle+f(x) \leq f(y)\right\} .
$$

For $\varepsilon \geq 0$, the $\varepsilon$-subdifferential of $f$ is defined by

$$
\partial_{\varepsilon} f: X \rightrightarrows X^{*}: x \mapsto\left\{x^{*} \in X^{*} \mid(\forall y \in X)\left\langle y-x, x^{*}\right\rangle+f(x) \leq f(y)+\varepsilon\right\} .
$$

Note that $\partial f=\partial_{0} f$. We denote by $J:=J_{X}$ the duality map, i.e., the subdifferential of the function $\frac{1}{2}\|\cdot\|^{2}$ mapping $X$ to $X^{*}$.

Now let $\left.\left.F: X \times X^{*} \rightarrow\right]-\infty,+\infty\right]$. We say $F$ is a $B C$-function (BC stands for "Bigger conjugate") [35] if $F$ is proper and convex with

$$
F^{*}\left(x^{*}, x\right) \geq F\left(x, x^{*}\right) \geq\left\langle x, x^{*}\right\rangle \quad \forall\left(x, x^{*}\right) \in X \times X^{*} .
$$

Let $Y$ be another real Banach space. We set $P_{X}: X \times Y \rightarrow X:(x, y) \mapsto x$, and $P_{Y}$ : $X \times Y \rightarrow Y:(x, y) \mapsto y$. Let $L: X \rightarrow Y$ be linear. We say $L$ is a (linear) isomorphism into $Y$ if $L$ is one to one, continuous and $L^{-1}$ is continuous on $\operatorname{ran} L$. We say $L$ is an isometry if $\|L x\|=\|x\|, \forall x \in X$. The spaces $X, Y$ are then isometric (isomorphic) if there exists an isometry (isomorphism) from $X$ onto $Y$.

Let $\left.\left.F_{1}, F_{2}: X \times Y \rightarrow\right]-\infty,+\infty\right]$. Then the partial inf-convolution $F_{1} \square_{1} F_{2}$ is the function defined on $X \times Y$ by

$$
F_{1} \square_{1} F_{2}:(x, y) \mapsto \inf _{u \in X} F_{1}(u, y)+F_{2}(x-u, y) .
$$

Then $F_{1} \square_{2} F_{2}$ is the function defined on $X \times Y$ by

$$
F_{1} \square_{2} F_{2}:(x, y) \mapsto \inf _{v \in Y} F_{1}(x, y-v)+F_{2}(x, v) .
$$

In Example 4.1](vi) \& (viii) of this paper, we provide a negative answer to the following question posed by S. Simons [35, Problem 22.12]: 
Let $\left.\left.F_{1}, F_{2}: X \times X^{*} \rightarrow\right]-\infty,+\infty\right]$ be proper lower semicontinuous and convex. Assume that $F_{1}, F_{2}$ are $B C$-functions and that

$$
\bigcup_{\lambda>0} \lambda\left[P_{X^{*}} \operatorname{dom} F_{1}-P_{X^{*}} \operatorname{dom} F_{2}\right] \text { is a closed subspace of } X^{*} .
$$

Is $F_{1} \square_{1} F_{2}$ necessarily a $B C$-function?

We are now ready to set to work. The paper is organized as follows. In Section 2, we collect auxiliary results for future reference and for the reader's convenience. Our main result (Theorem 3.6) is established in Section 3, In Section 4, we provide various applications and extensions including the promised negative answer to Simons' question. Furthermore, we show that every Banach space containing an isomorphic copy of the James space $\mathbf{J}$ or of $\mathbf{J}^{*}$, of $\ell^{1}$ or of $c_{0}$ is not of type (D) (Example 4.1](xi) or Corollary 4.12, Corollary 4.11 and Example 4.13).

\section{Auxiliary results}

Observation:

Fact 2.1 (See [26, Proposition 2.6.6(c)]). Let D be a subspace of $X^{*}$. Then $\left(D_{\perp}\right)^{\perp}=\bar{D}^{\mathrm{w}^{*}}$.

We now record a famous Banach space result:

Fact 2.2 (Banach and Mazur) (See [16, Theorem 5.8, page 240] or [15, Theorem 5.17, page 144]).) Every separable Banach space is isometric to a subspace of $C[0,1]$.

Now we turn to prerequisite results on Fitzpatrick functions, monotone operators, and linear relations.

Fact 2.3 (Fitzpatrick) (See [17, Corollary 3.9 and Proposition 4.2] and [7, 11].) Let $A: X \rightrightarrows X^{*}$ be maximally monotone, and set

$$
\left.\left.F_{A}: X \times X^{*} \rightarrow\right]-\infty,+\infty\right]:\left(x, x^{*}\right) \mapsto \sup _{\left(a, a^{*}\right) \in \operatorname{gra} A}\left(\left\langle x, a^{*}\right\rangle+\left\langle a, x^{*}\right\rangle-\left\langle a, a^{*}\right\rangle\right),
$$

which is the Fitzpatrick function associated with $A$. Then $F_{A}$ is a $B C$-function and $F_{A}=$ $\langle\cdot, \cdot\rangle$ on $\operatorname{gra} A$. 
Fact 2.4 (Simons and Zălinescu) (See [38, Theorem 4.2] or [35, Theorem 16.4(a)].) Let $Y$ be a real Banach space and $\left.\left.F_{1}, F_{2}: X \times Y \rightarrow\right]-\infty,+\infty\right]$ be proper, lower semicontinuous, and convex. Assume that for every $(x, y) \in X \times Y$,

$$
\left(F_{1} \square_{2} F_{2}\right)(x, y)>-\infty
$$

and that $\bigcup_{\lambda>0} \lambda\left[P_{X} \operatorname{dom} F_{1}-P_{X} \operatorname{dom} F_{2}\right]$ is a closed subspace of $X$. Then for every $\left(x^{*}, y^{*}\right) \in X^{*} \times Y^{*}$,

$$
\left(F_{1} \square_{2} F_{2}\right)^{*}\left(x^{*}, y^{*}\right)=\min _{u^{*} \in X^{*}}\left[F_{1}^{*}\left(x^{*}-u^{*}, y^{*}\right)+F_{2}^{*}\left(u^{*}, y^{*}\right)\right] .
$$

Fact 2.5 (Simons and Zălinescu) (See [35, Theorem 16.4(b)].) Let $Y$ be a real Banach space and $\left.\left.F_{1}, F_{2}: X \times Y \rightarrow\right]-\infty,+\infty\right]$ be proper, lower semicontinuous and convex. Assume that for every $(x, y) \in X \times Y$,

$$
\left(F_{1} \square_{1} F_{2}\right)(x, y)>-\infty
$$

and that $\bigcup_{\lambda>0} \lambda\left[P_{Y} \operatorname{dom} F_{1}-P_{Y} \operatorname{dom} F_{2}\right]$ is a closed subspace of $Y$. Then for every $\left(x^{*}, y^{*}\right) \in$ $X^{*} \times Y^{*}$,

$$
\left(F_{1} \square_{1} F_{2}\right)^{*}\left(x^{*}, y^{*}\right)=\min _{v^{*} \in Y^{*}}\left[F_{1}^{*}\left(x^{*}, v^{*}\right)+F_{2}^{*}\left(x^{*}, y^{*}-v^{*}\right)\right] .
$$

Phelps and Simons proved the next Fact 2.6 for unbounded linear operators in [29, Proposition 3.2(a)], but their proof can also be adapted for general linear relations. For reader's convenience, we write down their proof.

Fact 2.6 (Phelps and Simons) Let $A: X \rightrightarrows X^{*}$ be a monotone linear relation. Then $\left(x, x^{*}\right) \in X \times X^{*}$ is monotonically related to gra $A$ if and only if

$$
\left\langle x, x^{*}\right\rangle \geq 0 \text { and }\left[\left\langle y^{*}, x\right\rangle+\left\langle x^{*}, y\right\rangle\right]^{2} \leq 4\left\langle x^{*}, x\right\rangle\left\langle y^{*}, y\right\rangle, \quad \forall\left(y, y^{*}\right) \in \operatorname{gra} A .
$$

Proof. We have the following equivalences:

$\left(x, x^{*}\right) \in X \times X^{*}$ is monotonically related to gra $A$

$\Leftrightarrow \lambda^{2}\left\langle y, y^{*}\right\rangle-\lambda\left[\left\langle y^{*}, x\right\rangle+\left\langle x^{*}, y\right\rangle\right]+\left\langle x, x^{*}\right\rangle=\left\langle\lambda y^{*}-x^{*}, \lambda y-x\right\rangle \geq 0, \forall \lambda \in \mathbb{R}, \forall\left(y, y^{*}\right) \in \operatorname{gra} A$

$\Leftrightarrow\left\langle x, x^{*}\right\rangle \geq 0$ and $\left[\left\langle y^{*}, x\right\rangle+\left\langle x^{*}, y\right\rangle\right]^{2} \leq 4\left\langle x^{*}, x\right\rangle\left\langle y^{*}, y\right\rangle, \forall\left(y, y^{*}\right) \in$ gra $A$ (by [29, Lemma 2.1]).

This completes the proof.

Fact 2.7 (Simons / Marques Alves and Svaiter) (See [32, Lemma 15] or [35, Theorem 36.3(a)], and [25, Theorem 4.4].) Let $A: X \rightrightarrows X^{*}$ be maximally monotone. Then $A$ is of type (D) if and only if it is of type (NI). 
We next cite some properties regarding the uniqueness of (maximally) monotone extension of a maximally monotone operator to $X^{* *} \times X^{*}$. Simons showed that every maximally monotone operator of type (NI) is unique in [34]. Recently, Marques Alves and Svaiter contributed the following results:

Fact 2.8 (Marques Alves and Svaiter) (See [24, Theorem 1.6].) Let $A: X \rightrightarrows X^{*}$ be $a$ maximally monotone linear relation that is not of type (D). Assume that $A$ is unique. Then $\operatorname{gra} A=\operatorname{dom} F_{A}$.

Fact 2.9 (Marques Alves and Svaiter) (See [25, Corollary 4.6].) Let $A: X \rightrightarrows X^{*}$ be $a$ maximally monotone operator such that gra $A$ is not affine. Then $A$ is of type $(D)$ if and only if $A$ is unique.

The Gossez operator defined as in Example 4.1](xii) is a maximally monotone and unique operator that is not of type (D) [20].

The definition of operators of type (BR) directly yields the following result.

Fact 2.10 Let $A: X \rightrightarrows X^{*}$ be maximally monotone and $\left(x, x^{*}\right) \in X \times X^{*}$. Assume that $A$ is of type $(B R)$ and that $\inf _{\left(a, a^{*}\right) \in \operatorname{gra} A}\left\langle x-a, x^{*}-a^{*}\right\rangle>-\infty$. Then $x \in \overline{\operatorname{dom} A}$ and $x^{*} \in \overline{\operatorname{ran} A}$.

Additionally,

Fact 2.11 (Marques Alves and Svaiter) (See [24, Theorem 1.4(4)] or [23].) Let $A$ : $X \rightrightarrows X^{*}$ be a maximally monotone operator. Assume that $A$ is of type (NI). Then $A$ is of type $(B R)$.

We shall also need some precise results about linear relations. The first two are elementary.

Fact 2.12 (Cross) (See [14, Proposition I.2.8(a)].) Let $A: X \rightrightarrows Y$ be a linear relation. Then $\left(\forall\left(x, x^{*}\right) \in \operatorname{gra} A\right) A x=x^{*}+A 0$.

Lemma 2.13 Let $A: X \rightrightarrows X^{*}$ be a linear relation. Assume that $A^{*}$ is monotone. Then $\operatorname{ker} A^{*} \subseteq\left(\operatorname{ran} A^{*}\right)^{\perp}$.

Proof. Let $x^{* *} \in \operatorname{ker} A^{*}$ and then $\left(\alpha x^{* *}, 0\right) \in \operatorname{gra} A^{*}, \forall \alpha \in \mathbb{R}$. Then

$$
0 \leq\left\langle\alpha x^{* *}+y^{* *}, y^{*}\right\rangle=\alpha\left\langle x^{* *}, y^{*}\right\rangle+\left\langle y^{* *}, y^{*}\right\rangle, \quad \forall\left(y^{* *}, y^{*}\right) \in \operatorname{gra} A^{*}, \forall \alpha \in \mathbb{R} .
$$

Hence $\left\langle x^{* *}, y^{*}\right\rangle=0, \quad \forall\left(y^{* *}, y^{*}\right) \in \operatorname{gra} A^{*}$ and thus $x^{* *} \in\left(\operatorname{ran} A^{*}\right)^{\perp}$. Thus $\operatorname{ker} A^{*} \subseteq$ $\left(\operatorname{ran} A^{*}\right)^{\perp}$. 
Fact 2.14 (See [4, Theorem 3.1].) Let $A: X \rightrightarrows X^{*}$ be a maximally monotone linear relation. Then $A$ is of type $(D)$ if and only if $A^{*}$ is monotone.

Fact 2.15 (See [41, Theorem 3.1].) Let $A: X \rightrightarrows X^{*}$ be a maximally monotone linear relation, and let $f: X \rightarrow]-\infty,+\infty]$ be a proper lower semicontinuous convex function with $\operatorname{dom} A \cap \operatorname{int} \operatorname{dom} \partial f \neq \varnothing$. Then $A+\partial f$ is maximally monotone.

Fact 2.16 (Simons) (See [35, Theorem 28.9].) Let $Y$ be a Banach space, and $L: Y \rightarrow X$ be continuous and linear with $\operatorname{ran} L$ closed and $\operatorname{ran} L^{*}=Y^{*}$. Let $A: X \rightrightarrows X^{*}$ be monotone with $\operatorname{dom} A \subseteq \operatorname{ran} L$ such that gra $A \neq \varnothing$. Then $A$ is maximally monotone if, and only if $A$ is ran $L$-saturated and $L^{*} A L$ is maximally monotone.

Theorem 2.17 Let $Y$ be a Banach space, and $L: Y \rightarrow X$ be an isomorphism into $X$. Let $T: Y \rightrightarrows Y^{*}$ be monotone. Then $T$ is maximally monotone if, and only if $\left(L^{*}\right)^{-1} T L^{-1}$, mapping $X$ into $X^{*}$, is maximally monotone.

Proof. Let $A=\left(L^{*}\right)^{-1} T L^{-1}$. Then $\operatorname{dom} A \subseteq \operatorname{ran} L$. Since $L$ is an isomorphism into $X$, ran $L$ is closed. By [26, Theorem 3.1.22(b)] or [15, Exercise 2.39(i), page 59], ran $L^{*}=$ $Y^{*}$. Hence $\operatorname{gra}\left(L^{*}\right)^{-1} T L^{-1} \neq \varnothing$ if and only if gra $T \neq \varnothing$. Clearly, $A$ is monotone. Since $\{0\} \times(\operatorname{ran} L)^{\perp} \subseteq \operatorname{gra}\left(L^{*}\right)^{-1}$ and then by Fact 2.12, $A=\left(L^{*}\right)^{-1} T L^{-1}$ is ran $L$-saturated. By Fact 2.16, $A=\left(L^{*}\right)^{-1} T L^{-1}$ is maximally monotone if and only if $L^{*} A L=T$ is maximally monotone.

The following consequence will allow us to construct maximally monotone operators that are not of type (D) in a variety of non-reflexive Banach spaces.

Corollary 2.18 (Subspaces) Let $Y$ be a Banach space, and $L: Y \rightarrow X$ be an isomorphism into $X$. Let $T: Y \rightrightarrows Y^{*}$ be maximally monotone. Assume that $T$ is not of type $(D)$. Then $\left(L^{*}\right)^{-1} T L^{-1}$ is a maximally monotone operator mapping $X$ into $X^{*}$ that is not of type $(D)$. In particular, every Banach subspace of a type (D) space is of type (D).

Proof. By Theorem 2.17, $\left(L^{*}\right)^{-1} T L^{-1}$ is maximally monotone. By Fact 2.7, there exists $\left(y_{0}^{* *}, y_{0}^{*}\right) \in Y^{* *} \times Y^{*}$ such that

$$
\sup _{\left(b, b^{*}\right) \in \operatorname{gra} T}\left\{\left\langle y_{0}^{* *}, b^{*}\right\rangle+\left\langle y_{0}^{*}, b\right\rangle-\left\langle b, b^{*}\right\rangle\right\}<\left\langle y_{0}^{* *}, y_{0}^{*}\right\rangle
$$

By [26, Theorem 3.1.22(b)] or [15, Exercise 2.39(i), page 59], ran $L^{*}=Y^{*}$ and thus there exists $x_{0}^{*} \in X^{*}$ such that $L^{*} x_{0}^{*}=y_{0}^{*}$. Let $A=\left(L^{*}\right)^{-1} T L^{-1}$. Then we have

$$
\begin{aligned}
& \sup _{\left(a, a^{*}\right) \in \operatorname{gra} A}\left\{\left\langle L^{* *} y_{0}^{* *}, a^{*}\right\rangle+\left\langle x_{0}^{*}, a\right\rangle-\left\langle a, a^{*}\right\rangle\right\} \\
& =\sup _{\left(L y, a^{*}\right) \in \operatorname{gra} A}\left\{\left\langle y_{0}^{* *}, L^{*} a^{*}\right\rangle+\left\langle x_{0}^{*}, L y\right\rangle-\left\langle L y, a^{*}\right\rangle\right\}
\end{aligned}
$$




$$
\begin{aligned}
& =\sup _{\left(L y, a^{*}\right) \in \operatorname{gra} A}\left\{\left\langle y_{0}^{* *}, L^{*} a^{*}\right\rangle+\left\langle L^{*} x_{0}^{*}, y\right\rangle-\left\langle y, L^{*} a^{*}\right\rangle\right\} \\
& =\sup _{\left(L y, a^{*}\right) \in \operatorname{gra} A}\left\{\left\langle y_{0}^{* *}, L^{*} a^{*}\right\rangle+\left\langle y_{0}^{*}, y\right\rangle-\left\langle y, L^{*} a^{*}\right\rangle\right\} \\
& =\sup _{\left(y, y^{*}\right) \in \operatorname{gra} T}\left\{\left\langle y_{0}^{* *}, y^{*}\right\rangle+\left\langle y_{0}^{*}, y\right\rangle-\left\langle y, y^{*}\right\rangle\right\} \quad\left(\text { by }\left(L y, a^{*}\right) \in \operatorname{gra} A \Leftrightarrow\left(y, L^{*} a^{*}\right) \in \operatorname{gra} T\right) \\
& \left.<\left\langle y_{0}^{* *}, y_{0}^{*}\right\rangle \quad(\text { by (6) })\right) \\
& (7) \quad\left\langle L^{* *} y_{0}^{* *}, x_{0}^{*}\right\rangle .
\end{aligned}
$$

Thus $A$ is not of type (NI) and hence $A=\left(L^{*}\right)^{-1} T L^{-1}$ is not of type (D) by Fact 2.7.

Note that it follows that $X$ is of type (D) whenever $X^{* *}$ is.

\section{Main result}

We start with several technical tools. To relate Fitzpatrick functions and skew operators we have:

Lemma 3.1 Let $A: X \rightrightarrows X^{*}$ be a skew linear relation. Then

$$
F_{A}=\iota_{\mathrm{gra}\left(-A^{*}\right) \cap X \times X^{*}}
$$

Proof. Let $\left(x_{0}, x_{0}^{*}\right) \in X \times X^{*}$. We have

$$
\begin{aligned}
F_{A}\left(x_{0}, x_{0}^{*}\right) & =\sup _{\left(x, x^{*}\right) \in \operatorname{gra} A}\left\{\left\langle\left(x_{0}^{*}, x_{0}\right),\left(x, x^{*}\right)\right\rangle-\left\langle x, x^{*}\right\rangle\right\} \\
& =\sup _{\left(x, x^{*}\right) \in \operatorname{gra} A}\left\langle\left(x_{0}^{*}, x_{0}\right),\left(x, x^{*}\right)\right\rangle \\
& =\iota_{(\operatorname{gra} A)^{\perp}}\left(x_{0}^{*}, x_{0}\right) \\
& =\iota_{\operatorname{gra}\left(-A^{*}\right)}\left(x_{0}, x_{0}^{*}\right) \\
& =\iota_{\operatorname{gra}\left(-A^{*}\right) \cap X \times X^{*}}\left(x_{0}, x_{0}^{*}\right) .
\end{aligned}
$$

Hence (8) holds.

To produce operators not of type (D) but that are of (BR) we exploit:

Lemma 3.2 Let $A: X \rightrightarrows X^{*}$ be a maximally monotone and linear skew operator. Assume that gra $\left(-A^{*}\right) \cap X \times X^{*} \subseteq \operatorname{gra} A$. Then $A$ is of type (BR). 
Proof. Let $\alpha, \beta>0$ and $\left(x, x^{*}\right) \in X \times X^{*}$ be such that $\inf _{\left(a, a^{*}\right) \in \operatorname{gra} A}\left\langle x-a, x^{*}-a^{*}\right\rangle>-\alpha \beta$. Since $A$ is skew, we have

$$
\inf _{\left(a, a^{*}\right) \in \operatorname{gra} A}\left\langle x, x^{*}\right\rangle-\left[\left\langle x, a^{*}\right\rangle+\left\langle a, x^{*}\right\rangle\right]=\inf _{\left(a, a^{*}\right) \in \operatorname{gra} A}\left\langle x-a, x^{*}-a^{*}\right\rangle>-\alpha \beta .
$$

Thus, $\left\langle x, a^{*}\right\rangle+\left\langle a, x^{*}\right\rangle=0, \forall\left(a, a^{*}\right) \in \operatorname{gra} A$ and hence $\left(x, x^{*}\right) \in \operatorname{gra}\left(-A^{*}\right)$. Then by assumption, $\left(x, x^{*}\right) \in \operatorname{gra} A$. Taking $\left(b, b^{*}\right)=\left(x, x^{*}\right)$, we have $\|b-x\|<\alpha$ and $\left\|b^{*}-x^{*}\right\|<\beta$. Hence $A$ is of type (BR).

Corollary 3.3 Let $A: X \rightrightarrows X^{*}$ be a maximally monotone and linear skew operator that is not of type (D). Assume that $A$ is unique. Then gra $A=\operatorname{gra}\left(-A^{*}\right) \cap X \times X^{*}$ and so $A$ is of type $(B R)$.

Proof. Apply Fact 2.8, Lemma 3.1 and Lemma 3.2 directly.

Proposition 3.4 Let $A: X \rightrightarrows X^{*}$ be maximally monotone. Assume that $A$ is of type (NI) and that there exists $e \in X^{*}$ such that

$$
\left\langle x^{*}, x\right\rangle \geq\langle e, x\rangle^{2}, \quad \forall\left(x, x^{*}\right) \in \operatorname{gra} A .
$$

Then $e \in \overline{\operatorname{conv} \operatorname{ran} A}$.

Proof. Suppose $e \notin \overline{\operatorname{conv} \operatorname{ran} A}$. Then by the Separation Theorem, there exists $x_{0}^{* *} \in X^{* *}$ such that $\left\langle e-x^{*}, x_{0}^{* *}\right\rangle \geq 1$ for all $x^{*} \in \operatorname{ran} A$. Then we have

$$
\begin{aligned}
\left\langle x^{*}-e, x-x_{0}^{* *}\right\rangle & =\left\langle e-x^{*}, x_{0}^{* *}\right\rangle+\left\langle x^{*}-e, x\right\rangle, \quad \forall\left(x, x^{*}\right) \in \operatorname{gra} A \\
& \geq 1+\langle e, x\rangle^{2}-\langle e, x\rangle \\
& \geq \min _{t \in \mathbb{R}} t^{2}-t+1=\frac{3}{4} .
\end{aligned}
$$

Thus $A$ is not of type (NI), which contradicts the assumption.

The proof of the following result was partially inspired by that [12, Proposition 2.2].

Proposition 3.5 Let $A: X \rightrightarrows X^{*}$ be a maximally monotone linear relation. Assume that there exists $e \in X^{*}$ such that $e \notin \overline{\operatorname{ran} A}$ and that

$$
\left\langle x^{*}, x\right\rangle \geq\langle e, x\rangle^{2}, \quad \forall\left(x, x^{*}\right) \in \operatorname{gra} A .
$$

Then $A$ is neither of type (D) nor unique. 
Proof. By Proposition 3.4, $A$ is not of type (NI) and hence $A$ is not of type (D) by Fact 2.7. Similar to the proof of Proposition 3.4, there exists $x_{0}^{* *} \in X^{* *}$ such that $\left\langle e, x_{0}^{* *}\right\rangle \geq 1$ and $x_{0}^{* *} \in(\operatorname{ran} A)^{\perp}$. Let $0<\alpha<2$. Then we have

$$
\begin{aligned}
\left\langle x^{*}-\alpha e, x-\frac{1}{\alpha} x_{0}^{* *}\right\rangle & =\left\langle\alpha e-x^{*}, \frac{1}{\alpha} x_{0}^{* *}\right\rangle+\left\langle x^{*}-\alpha e, x\right\rangle, \quad \forall\left(x, x^{*}\right) \in \operatorname{gra} A \\
& \geq 1+\langle e, x\rangle^{2}-\alpha\langle e, x\rangle \\
& \geq \min _{t \in \mathbb{R}} t^{2}-\alpha t+1 \\
& =1-\frac{\alpha^{2}}{4}>0 .
\end{aligned}
$$

Thus for every $0<\alpha<2,\left(\frac{1}{\alpha} x_{0}^{* *}, \alpha e\right) \in X^{* *} \times X^{*}$ is monotonically related to gra $A$. Take $0<\alpha_{1}<\alpha_{2}<2$. Then by Zorn's Lemma, we have a maximally monotone extension, $A_{1}: X^{* *} \rightrightarrows X^{*}$ such that gra $A_{1} \supseteq \operatorname{gra} A \cup\left\{\left(\frac{1}{\alpha_{1}} x_{0}^{* *}, \alpha_{1} e,\right)\right\}$, and we can also obtain a maximally monotone extension, $A_{2}: X^{* *} \rightrightarrows X^{*}$ such that gra $A_{2} \supseteq \operatorname{gra} A \cup\left\{\left(\frac{1}{\alpha_{2}} x_{0}^{* *}, \alpha_{2} e\right)\right\}$.

Now we show gra $A_{1} \neq \operatorname{gra} A_{2}$. Suppose to the contrary that gra $A_{1}=\operatorname{gra} A_{2}$. Then by the monotonicity of $A_{1}$, we have

$$
\left\langle\frac{1}{\alpha_{1}} x_{0}^{* *}-\frac{1}{\alpha_{2}} x_{0}^{* *}, \alpha_{1} e-\alpha_{2} e\right\rangle \geq 0 .
$$

On the other hand,

$$
\begin{aligned}
\left\langle\frac{1}{\alpha_{1}} x_{0}^{* *}-\frac{1}{\alpha_{2}} x_{0}^{* *}, \alpha_{1} e-\alpha_{2} e\right\rangle & =\left(\alpha_{1}-\alpha_{2}\right)\left(\frac{1}{\alpha_{1}}-\frac{1}{\alpha_{2}}\right)\left\langle x_{0}^{* *}, e\right\rangle \\
& <\left(\alpha_{1}-\alpha_{2}\right)\left(\frac{1}{\alpha_{1}}-\frac{1}{\alpha_{2}}\right)<0,
\end{aligned}
$$

which contradicts (10). Hence gra $A_{1} \neq \operatorname{gra} A_{2}$ and thus $A$ is not unique.

We are now ready to establish our work-horse Theorem [3.6, which allows us to construct various maximally monotone operators — both linear and nonlinear — that are not of type (D). The idea of constructing the operators in the following fashion is based upon [2, Theorem 5.1] and was stimulated by [12].

Theorem 3.6 (Predual constructions) Let $A: X^{*} \rightarrow X^{* *}$ be linear and continuous. Assume that $\operatorname{ran} A \subseteq X$ and that there exists $e \in X^{* *} \backslash X$ such that

$$
\left\langle A x^{*}, x^{*}\right\rangle=\left\langle e, x^{*}\right\rangle^{2}, \quad \forall x^{*} \in X^{*} .
$$

Let $P$ and $S$ respectively be the symmetric part and antisymmetric part of $A$. Let $T: X \rightrightarrows X^{*}$ be defined by

$$
\begin{aligned}
\operatorname{gra} T & :=\left\{\left(-S x^{*}, x^{*}\right) \mid x^{*} \in X^{*},\left\langle e, x^{*}\right\rangle=0\right\} \\
& =\left\{\left(-A x^{*}, x^{*}\right) \mid x^{*} \in X^{*},\left\langle e, x^{*}\right\rangle=0\right\} .
\end{aligned}
$$

Let $f: X \rightarrow]-\infty,+\infty]$ be a proper lower semicontinuous and convex function. Set $F:=$ $f \oplus f^{*}$ on $X \times X^{*}$. Then the following hold. 
(i) A is a maximally monotone operator on $X^{*}$ that is neither of type (D) nor unique.

(ii) $P x^{*}=\left\langle x^{*}, e\right\rangle e, \forall x^{*} \in X^{*}$.

(iii) $T$ is maximally monotone and skew on $X$.

(iv) $\operatorname{gra} T^{*}=\left\{\left(S x^{*}+r e, x^{*}\right) \mid x^{*} \in X^{*}, r \in \mathbb{R}\right\}$.

(v) $-T$ is not maximally monotone.

(vi) $T$ is not of type $(D)$.

(vii) $F_{T}=\iota_{C}$, where

$$
C:=\left\{\left(-A x^{*}, x^{*}\right) \mid x^{*} \in X^{*}\right\}
$$

(viii) $T$ is not unique.

(ix) $T$ is not of type (BR).

(x) If $\operatorname{dom} T \cap \operatorname{int} \operatorname{dom} \partial f \neq \varnothing$, then $T+\partial f$ is maximally monotone.

(xi) $F$ and $F_{T}$ are $B C$-functions on $X \times X^{*}$.

(xii) Moreover,

$$
\bigcup_{\lambda>0} \lambda\left(P_{X^{*}}\left(\operatorname{dom} F_{T}\right)-P_{X^{*}}(\operatorname{dom} F)\right)=X^{*}
$$

while, assuming that there exists $\left(v_{0}, v_{0}^{*}\right) \in X \times X^{*}$ such that

$$
f^{*}\left(v_{0}^{*}\right)+f^{* *}\left(v_{0}-A^{*} v_{0}^{*}\right)<\left\langle v_{0}, v_{0}^{*}\right\rangle,
$$

then $F_{T} \square_{1} F$ is not a $B C$-function.

(xiii) Assume that $\left[\operatorname{ran} A-\bigcup_{\lambda>0} \lambda \operatorname{dom} f\right]$ is a closed subspace of $X$ and that

$$
\varnothing \neq\left.\operatorname{dom} f^{* *} \circ A^{*}\right|_{X^{*}} \nsubseteq\{e\}_{\perp} .
$$

Then $T+\partial f$ is not of type $(D)$.

(xiv) Assume that $\operatorname{dom} f^{* *}=X^{* *}$. Then $T+\partial f$ is a maximally monotone operator that is not of type (D). 
Proof. (i). Clearly, $A$ has full domain. Since $A$ is monotone and continuous, $A$ is maximally monotone. By the assumptions that $e \notin X$ and $\overline{\operatorname{ran} A} \subseteq \bar{X}=X$, then by Proposition 3.5, $A$ is neither of type (D) nor unique. See also [1, Theorem 14.2.1 and Theorem 13.2.3] for alternative proof of that $A$ is not of type (D).

(ii): Now we show that

$$
P x^{*}=\left\langle x^{*}, e\right\rangle e, \forall x^{*} \in X^{*} .
$$

Since $\langle\cdot, e\rangle e=\partial\left(\frac{1}{2}\langle\cdot, e\rangle^{2}\right)$ and by [29, Theorem 5.1], $\langle\cdot, e\rangle e$ is a symmetric operator on $X^{*}$. Clearly, $A-\langle\cdot, e\rangle e$ is skew. Then (14) holds.

(iii): Let $x^{*} \in X^{*}$ with $\left\langle e, x^{*}\right\rangle=0$. Then we have

$$
S x^{*}=\left\langle x^{*}, e\right\rangle e+S x^{*}=P x^{*}+S x^{*}=A x^{*} \in \operatorname{ran} A \subseteq X .
$$

Thus (11) holds and $T$ is well defined.

We have $S$ is skew and hence $T$ is skew. Let $\left(z, z^{*}\right) \in X \times X^{*}$ be monotonically related to gra T. By Fact 2.6, we have

$$
0=\left\langle z, x^{*}\right\rangle+\left\langle-S x^{*}, z^{*}\right\rangle=\left\langle z+S z^{*}, x^{*}\right\rangle, \quad \forall x^{*} \in\{e\}_{\perp} .
$$

Thus by Fact 2.1, we have $z+S z^{*} \in\left(\{e\}_{\perp}\right)^{\perp}=\operatorname{span}\{e\}$ and then

$$
z=-S z^{*}+\kappa e, \exists \kappa \in \mathbb{R} .
$$

By $(0,0) \in \operatorname{gra} T$,

$$
\kappa\left\langle z^{*}, e\right\rangle=\left\langle-S z^{*}+\kappa e, z^{*}\right\rangle=\left\langle z, z^{*}\right\rangle \geq 0 .
$$

Then by (15) and (ii),

$$
A z^{*}=P z^{*}+S z^{*}=P z^{*}+\kappa e-z=\left[\left\langle z^{*}, e\right\rangle+\kappa\right] e-z .
$$

By the assumptions that $z \in X, A z^{*} \in X$ and $e \notin X,\left[\left\langle z^{*}, e\right\rangle+\kappa\right]=0$ by (17). Then by (16), we have $\left\langle z^{*}, e\right\rangle=\kappa=0$ and thus $\left(z, z^{*}\right) \in \operatorname{gra} T$ by (15). Hence $T$ is maximally monotone.

(iv): Let $\left(x_{0}^{* *}, x_{0}^{*}\right) \in X^{* *} \times X^{*}$. Then we have

$$
\begin{aligned}
& \left(x_{0}^{* *}, x_{0}^{*}\right) \in \operatorname{gra} T^{*} \Leftrightarrow\left\langle x_{0}^{*}, S x^{*}\right\rangle+\left\langle x^{*}, x_{0}^{* *}\right\rangle=0, \quad \forall x^{*} \in\{e\}_{\perp} \\
& \Leftrightarrow\left\langle x^{*}, x_{0}^{* *}-S x_{0}^{*}\right\rangle=0, \quad \forall x^{*} \in\{e\}_{\perp} \\
& \Leftrightarrow x_{0}^{* *}-S x_{0}^{*} \in\left(\{e\}_{\perp}\right)^{\perp}=\operatorname{span}\{e\} \quad \text { (by Fact 2.1) } \\
& \Leftrightarrow x_{0}^{* *}-S x_{0}^{*}=r e, \quad \exists r \in \mathbb{R} .
\end{aligned}
$$


Thus gra $T^{*}=\left\{\left(S x^{*}+r e, x^{*}\right) \mid x^{*} \in X^{*}, r \in \mathbb{R}\right\}$.

$(\mathrm{v})$. Since $e \notin X$, we have $e \neq 0$. Then there exists $z^{*} \in X^{*}$ such that $z^{*} \notin\{e\}_{\perp}$. Then by (ii) (iv) and the assumption that $\operatorname{ran} A \subseteq X$, we have

$$
\left(A z^{*}, z^{*}\right)=\left(S z^{*}+\left\langle e, z^{*}\right\rangle e, x^{*}\right) \in \operatorname{gra} T^{*} \cap X \times X^{*} .
$$

Thus we have

$$
\begin{aligned}
\left\langle A z^{*}-x, z^{*}-x^{*}\right\rangle & =\left\langle A z^{*}, z^{*}\right\rangle-\left[\left\langle A z^{*}, x^{*}\right\rangle+\left\langle x, z^{*}\right\rangle\right]+\left\langle x, x^{*}\right\rangle \\
& =\left\langle A z^{*}, z^{*}\right\rangle \geq 0, \quad \forall\left(x, x^{*}\right) \in \operatorname{gra}(-T) .
\end{aligned}
$$

Hence $\left(A z^{*}, z^{*}\right)$ is monotonically related to $\operatorname{gra}(-T)$. Since $z^{*} \notin \operatorname{ran}(-T),\left(A z^{*}, z^{*}\right) \notin$ $\operatorname{gra}(-T)$ and then $-T$ is not maximally monotone.

(vi): By (iv), $T^{*}$ is not monotone. Then by Fact 2.14, $T$ is not of type (D).

(vii); By (iv), we have

$$
\begin{aligned}
& \left(z, z^{*}\right) \in \operatorname{gra}\left(-T^{*}\right) \cap X \times X^{*} \\
& \Leftrightarrow\left(z, z^{*}\right)=\left(-S z^{*}-r e, z^{*}\right), \quad z \in X, \exists r \in \mathbb{R}, z^{*} \in X^{*} \\
& \Leftrightarrow\left(z, z^{*}\right)=\left(-S z^{*}-\left\langle z^{*}, e\right\rangle e+\left[\left\langle z^{*}, e\right\rangle-r\right] e, z^{*}\right), \quad z \in X, \exists r \in \mathbb{R}, z^{*} \in X^{*} \\
& \Leftrightarrow\left(z, z^{*}\right)=\left(-A z^{*}+\left[\left\langle z^{*}, e\right\rangle-r\right] e, z^{*}\right), \quad z \in X, \exists r \in \mathbb{R}, z^{*} \in X^{*}(\text { by (ii) }) \\
& \Leftrightarrow\left(z, z^{*}\right)=\left(-A z^{*}, z^{*}\right), \quad\left\langle z^{*}, e\right\rangle=r\left(\text { since } z, A z^{*} \in X \text { and } e \notin X\right), \exists r \in \mathbb{R}, z^{*} \in X^{*} \\
& \Leftrightarrow\left(z, z^{*}\right) \in\left\{\left(-A x^{*}, x^{*}\right) \mid x^{*} \in X^{*}\right\}=C .
\end{aligned}
$$

Thus by Lemma 3.1, we have $F_{T}=\iota_{C}$.

(viii): Since $e \notin X$, we have $e \neq 0$. Then there exists $z^{*} \in X^{*}$ such that $z^{*} \notin\{e\}_{\perp}$. Thus $z^{*} \notin \operatorname{ran} T$. By (vii), $z^{*} \in P_{X^{*}}\left[\operatorname{dom} F_{T}\right]$. Thus, gra $T \neq \operatorname{dom} F_{T}$. Then by (vi)] and Fact 2.8, $T$ is not unique.

(ix) Suppose to the contrary that $T$ is of type (BR). Let $z^{*}$ be as in the proof of (viii). Then by Lemma 3.1 and (vii), we have $\left(-A z^{*}, z^{*}\right) \in \operatorname{gra}\left(-T^{*}\right) \cap X \times X^{*}$ and then

$$
\inf _{\left(a, a^{*}\right) \in \operatorname{gra} T}\left\langle-A z^{*}-a, z^{*}-a^{*}\right\rangle=\left\langle-A z^{*}, z^{*}\right\rangle>-\infty .
$$

Then Fact 2.10 shows $z^{*} \in \overline{\operatorname{ran} T}$, which contradicts that $z^{*} \notin\{e\}_{\perp}=\overline{\operatorname{ran} T}$. Hence $T$ is not of type (BR).

(x). Apply (iii) and Fact 2.15.

(xi) Clearly, $F$ is a BC-function. By (iii) and Fact 2.3, we see that $F_{T}$ is a BC-function. 
(xii): By (vii), we have

$$
\bigcup_{\lambda>0} \lambda\left(P_{X^{*}}\left(\operatorname{dom} F_{T}\right)-P_{X^{*}}(\operatorname{dom} F)\right)=X^{*}
$$

Then for every $\left(x, x^{*}\right) \in X \times X^{*}$ and $u \in X$, by (xi),

$$
F_{T}\left(x-u, x^{*}\right)+F\left(u, x^{*}\right)=F_{T}\left(x-u, x^{*}\right)+\left(f \oplus f^{*}\right)\left(u, x^{*}\right) \geq\left\langle x-u, x^{*}\right\rangle+\left\langle u, x^{*}\right\rangle=\left\langle x, x^{*}\right\rangle .
$$

Hence

$$
\left(F_{T} \square_{1} F\right)\left(x, x^{*}\right) \geq\left\langle x, x^{*}\right\rangle>-\infty .
$$

Then by (18), (19) and Fact 2.5,

$$
\begin{aligned}
\left(F_{T} \square_{1} F\right)^{*}\left(v_{0}^{*}, v_{0}\right) & =\min _{x^{* *} \in X^{* *}} F_{T}^{*}\left(v_{0}^{*}, x^{* *}\right)+F^{*}\left(v_{0}^{*}, v_{0}-x^{* *}\right) \\
& \leq F_{T}^{*}\left(v_{0}^{*}, A^{*} v_{0}^{*}\right)+F^{*}\left(v_{0}^{*}, v_{0}-A^{*} v_{0}^{*}\right) \\
& =0+F^{*}\left(v_{0}^{*}, v_{0}-A^{*} v_{0}^{*}\right) \quad(\text { by (vii) }) \\
& =\left(f \oplus f^{*}\right)^{*}\left(v_{0}^{*}, v_{0}-A^{*} v_{0}^{*}\right)=\left(f^{*} \oplus f^{* *}\right)\left(v_{0}^{*}, v_{0}-A^{*} v_{0}^{*}\right) \\
& =f^{*}\left(v_{0}^{*}\right)+f^{* *}\left(v_{0}-A^{*} v_{0}^{*}\right) \\
& <\left\langle v_{0}^{*}, v_{0}\right\rangle \quad(\text { by (13) }) .
\end{aligned}
$$

Hence $F_{T} \square_{1} F$ is not a BC-function.

(xiii); By the assumption, there exists $\left.x_{0}^{*} \in \operatorname{dom} f^{* *} \circ A^{*}\right|_{X^{*}}$ such that $\left\langle e, x_{0}^{*}\right\rangle \neq 0$. Let $\varepsilon_{0}=\frac{\left\langle e, x_{0}^{*}\right\rangle^{2}}{2}$. By [42, Theorem 2.4.4(iii)]), there exists $y_{0}^{* * *} \in \partial_{\varepsilon_{0}} f^{* *}\left(A^{*} x_{0}^{*}\right)$. By [42, Theorem 2.4.2(ii)]),

$$
f^{* *}\left(A^{*} x_{0}^{*}\right)+f^{* * *}\left(y_{0}^{* * *}\right) \leq\left\langle A^{*} x_{0}^{*}, y_{0}^{* * *}\right\rangle+\varepsilon_{0} .
$$

Then by [35, Lemma 45.9] or the proof of [30, Eq.(2.5) in Proposition 1], there exists $y_{0}^{*} \in X^{*}$ such that

$$
f^{* *}\left(A^{*} x_{0}^{*}\right)+f^{*}\left(y_{0}^{*}\right)<\left\langle A^{*} x_{0}^{*}, y_{0}^{*}\right\rangle+2 \varepsilon_{0} .
$$

Let $z_{0}^{*}=y_{0}^{*}+x_{0}^{*}$. Then by (21), we have

$$
\begin{aligned}
f^{* *}\left(A^{*} x_{0}^{*}\right)+f^{*}\left(z_{0}^{*}-x_{0}^{*}\right) & <\left\langle A^{*} x_{0}^{*}, z_{0}^{*}-x_{0}^{*}\right\rangle+2 \varepsilon_{0} \\
& =\left\langle A^{*} x_{0}^{*}, z_{0}^{*}\right\rangle-\left\langle A^{*} x_{0}^{*}, x_{0}^{*}\right\rangle+2 \varepsilon_{0} \\
& =\left\langle A^{*} x_{0}^{*}, z_{0}^{*}\right\rangle-\left\langle x_{0}^{*}, A x_{0}^{*}\right\rangle+2 \varepsilon_{0} \\
& =\left\langle A^{*} x_{0}^{*}, z_{0}^{*}\right\rangle-2 \varepsilon_{0}+2 \varepsilon_{0} \\
& =\left\langle A^{*} x_{0}^{*}, z_{0}^{*}\right\rangle .
\end{aligned}
$$


Then for every $\left(x, x^{*}\right) \in X \times X^{*}$ and $u^{*} \in X$, by (xi),

$F_{T}\left(x, x^{*}-u^{*}\right)+F\left(x, u^{*}\right)=F_{T}\left(x, x^{*}-u^{*}\right)+\left(f \oplus f^{*}\right)\left(x, u^{*}\right) \geq\left\langle x, x^{*}-u^{*}\right\rangle+\left\langle x, u^{*}\right\rangle=\left\langle x, x^{*}\right\rangle$.

Hence

$$
\left(F_{T} \square_{2} F\right)\left(x, x^{*}\right) \geq\left\langle x, x^{*}\right\rangle>-\infty .
$$

Then by (23), (vii) and Fact 2.4,

$$
\begin{aligned}
\left(F_{T} \square_{2} F\right)^{*}\left(z_{0}^{*}, A^{*} x_{0}^{*}\right) & =\min _{y^{*} \in X^{*}} F_{T}^{*}\left(y^{*}, A^{*} x_{0}^{*}\right)+F^{*}\left(z_{0}^{*}-y^{*}, A^{*} x_{0}^{*}\right) \\
& \leq F_{T}^{*}\left(x_{0}^{*}, A^{*} x_{0}^{*}\right)+F^{*}\left(z_{0}^{*}-x_{0}^{*}, A^{*} x_{0}^{*}\right) \\
& =0+F^{*}\left(z_{0}^{*}-x_{0}^{*}, A^{*} x_{0}^{*}\right) \quad(\text { by (vii) }) \\
& =\left(f \oplus f^{*}\right)^{*}\left(z_{0}^{*}-x_{0}^{*}, A^{*} x_{0}^{*}\right) \\
& =f^{*}\left(z_{0}^{*}-x_{0}^{*}\right)+f^{* *}\left(A^{*} x_{0}^{*}\right) \\
& <\left\langle z_{0}^{*}, A^{*} x_{0}^{*}\right\rangle \quad(\text { by (22) }) .
\end{aligned}
$$

Let $\left.\left.F_{0}: X \times X^{*} \rightarrow\right]-\infty,+\infty\right]$ be defined by

$$
\left(x, x^{*}\right) \mapsto\left\langle x, x^{*}\right\rangle+\iota_{\mathrm{gra}(T+\partial f)}\left(x, x^{*}\right) .
$$

Clearly, $F_{T} \square_{2} F \leq F_{0}$ on $X \times X^{*}$ and thus $\left(F_{T} \square_{2} F\right)^{*} \geq F_{0}^{*}$ on $X^{*} \times X^{* *}$. By (24), $F_{0}^{*}\left(z_{0}^{*}, A^{*} x_{0}^{*}\right)<\left\langle z_{0}^{*}, A^{*} x_{0}^{*}\right\rangle$. Hence $T+\partial f$ is not of type (NI) and thus $T+\partial f$ is not of type (D) by Fact 2.7 .

(xiv). Since $\operatorname{dom} f^{* *}=X^{* *}, \operatorname{dom} f=X$ by [42, Theorem 2.3.3]. By $\operatorname{dom} f^{* *}=X^{* *}$ again, $\left.\operatorname{dom} f^{* *} \circ A_{\alpha}^{*}\right|_{X^{*}}=X^{*} \nsubseteq\{\alpha\}_{\perp}$. Then apply (x) $\&$ (xiii) directly.

Remark 3.7 (Grothendieck spaces [11]) In light of part (xiii) of the previous theorem), we record that for a closed convex function

$$
\operatorname{dom} f=X \text { implies } \operatorname{dom} f^{* *}=X^{* *} \Leftrightarrow X \text { is a Grothendieck space. }
$$

All reflexive spaces are Grothendieck spaces while all non-reflexive Grothendieck spaces (such as $\left.L^{\infty}[0,1]\right)$ contain an isomorphic copy of $c_{0}$.

We are now ready to exploit Theorem 3.6.

\section{Examples and applications}

We begin with the case of $c_{0}$ and its dual $\ell^{1}$. 


\subsection{Applications to $c_{0}$}

Example $4.1\left(c_{0}\right)$ Let $X:=c_{0}$, with norm $\|\cdot\|_{\infty}$ so that $X^{*}=\ell^{1}$ with norm $\|\cdot\|_{1}$, and $X^{* *}=$ $\ell^{\infty}$ with its second dual norm $\|\cdot\|_{*}$. Let $\alpha:=\left(\alpha_{n}\right)_{n \in \mathbb{N}} \in \ell^{\infty}$ with $\lim \sup \alpha_{n} \neq 0$, and let $A_{\alpha}: \ell^{1} \rightarrow \ell^{\infty}$ be defined by

$$
\left(A_{\alpha} x^{*}\right)_{n}:=\alpha_{n}^{2} x_{n}^{*}+2 \sum_{i>n} \alpha_{n} \alpha_{i} x_{i}^{*}, \quad \forall x^{*}=\left(x_{n}^{*}\right)_{n \in \mathbb{N}} \in \ell^{1} .
$$

Now let $P_{\alpha}$ and $S_{\alpha}$ respectively be the symmetric part and antisymmetric part of $A_{\alpha}$. Let $T_{\alpha}: c_{0} \rightrightarrows X^{*}$ be defined by

$$
\begin{aligned}
\operatorname{gra} T_{\alpha} & :=\left\{\left(-S_{\alpha} x^{*}, x^{*}\right) \mid x^{*} \in X^{*},\left\langle\alpha, x^{*}\right\rangle=0\right\} \\
& =\left\{\left(-A_{\alpha} x^{*}, x^{*}\right) \mid x^{*} \in X^{*},\left\langle\alpha, x^{*}\right\rangle=0\right\} \\
& =\left\{\left(\left(-\sum_{i>n} \alpha_{n} \alpha_{i} x_{i}^{*}+\sum_{i<n} \alpha_{n} \alpha_{i} x_{i}^{*}\right)_{n}, x^{*}\right) \mid x^{*} \in X^{*},\left\langle\alpha, x^{*}\right\rangle=0\right\} .
\end{aligned}
$$

Then

(i) $\left\langle A_{\alpha} x^{*}, x^{*}\right\rangle=\left\langle\alpha, x^{*}\right\rangle^{2}, \quad \forall x^{*}=\left(x_{n}^{*}\right)_{n \in \mathbb{N}} \in \ell^{1}$ and(27) is well defined.

(ii) $A_{\alpha}$ is a maximally monotone operator on $\ell^{1}$ that is neither of type (D) nor unique.

(iii) $T_{\alpha}$ is a maximally monotone operator on $c_{0}$ that is not of type (D).

(iv) $-T_{\alpha}$ is not maximally monotone.

(v) $T_{\alpha}$ is neither unique nor of type (BR).

(vi) $F_{T_{\alpha}} \square_{1}\left(\|\cdot\| \oplus \iota_{B_{X^{*}}}\right)$ is not a BC-function.

(vii) $T_{\alpha}+\partial\|\cdot\|$ is a maximally monotone operator on $c_{0}(\mathbb{N})$ that is not of type (D).

(viii) If $\frac{1}{\sqrt{2}}<\|\alpha\|_{*} \leq 1$, then $F_{T_{\alpha}} \square_{1}\left(\frac{1}{2}\|\cdot\|^{2} \oplus \frac{1}{2}\|\cdot\|_{1}^{2}\right)$ is not a BC-function.

(ix) For $\lambda>0, T_{\alpha}+\lambda J$ is a maximally monotone operator on $c_{0}$ that is not of type (D).

(x) Let $\lambda>0$ and a linear isometry $L$ mapping $c_{0}$ to a subspace of $C[0,1]$ be given. Then both $\left(L^{*}\right)^{-1}\left(T_{\alpha}+\partial\|\cdot\|\right) L^{-1}$ and $\left(L^{*}\right)^{-1}\left(T_{\alpha}+\lambda J\right) L^{-1}$ are maximally monotone operators that are not of type (D). Hence neither $c_{0}$ nor $C[0,1]$ is of type (D).

(xi) Every Banach space that contains an isomorphic copy of $c_{0}$ is not of type (D). 
(xii) Let $G: \ell^{1} \rightarrow \ell^{\infty}$ be Gossez's operator [20] defined by

$$
\left(G\left(x^{*}\right)\right)_{n}:=\sum_{i>n} x_{i}^{*}-\sum_{i<n} x_{i}^{*}, \quad \forall\left(x_{n}^{*}\right)_{n \in \mathbb{N}} \in \ell^{1} .
$$

Then $T_{e}: c_{0} \rightrightarrows \ell^{1}$ as defined by

$$
\operatorname{gra} T_{e}:=\left\{\left(-G\left(x^{*}\right), x^{*}\right) \mid x^{*} \in \ell^{1},\left\langle x^{*}, e\right\rangle=0\right\}
$$

is a maximally monotone operator that is not of type $(\mathrm{D})$, where $e:=(1,1, \ldots, 1, \ldots)$.

(xiii) Moreover, $G$ is a unique maximally monotone operator that is not of type (D), but $G$ is of type $(\mathrm{BR})$.

Proof. We have $\alpha \notin c_{0}$. Since $\alpha=\left(\alpha_{n}\right)_{n \in \mathbb{N}} \in \ell^{\infty}$ and $\left\|A_{\alpha}\right\| \leq 2\|\alpha\|^{2}, A_{\alpha}$ is linear and continuous and $\operatorname{ran} A_{\alpha} \subseteq c_{0} \subseteq \ell^{\infty}$.

(i): We have

$$
\begin{aligned}
\left\langle A_{\alpha} x^{*}, x^{*}\right\rangle & =\sum_{n} x_{n}^{*}\left(\alpha_{n}^{2} x_{n}^{*}+2 \sum_{i>n} \alpha_{n} \alpha_{i} x_{i}^{*}\right) \\
& =\sum_{n} \alpha_{n}^{2} x_{n}^{* 2}+2 \sum_{n} \sum_{i>n} \alpha_{n} \alpha_{i} x_{n}^{*} x_{i}^{*} \\
& =\sum_{n} \alpha_{n}^{2} x_{n}^{* 2}+\sum_{n \neq i} \alpha_{n} \alpha_{i} x_{n}^{*} x_{i}^{*} \\
& =\left(\sum_{n} \alpha_{n} x_{n}^{*}\right)^{2}=\left\langle\alpha, x^{*}\right\rangle^{2}, \quad \forall x^{*}=\left(x_{n}^{*}\right)_{n \in \mathbb{N}} \in \ell^{1} .
\end{aligned}
$$

Then Theorem 3.6)(ii) shows that the symmetric part $P_{\alpha}$ of $A_{\alpha}$ is $P_{\alpha} x^{*}=\left\langle\alpha, x^{*}\right\rangle \alpha$ (for every $\left.x^{*} \in \ell^{1}\right)$. Thus, the skew part $S_{\alpha}$ of $A_{\alpha}$ is

$$
\begin{aligned}
\left(S_{\alpha} x^{*}\right)_{n} & =\left(A_{\alpha} x^{*}\right)_{n}-\left(P_{\alpha} x^{*}\right)_{n} \\
& =\alpha_{n}^{2} x_{n}^{*}+2 \sum_{i>n} \alpha_{n} \alpha_{i} x_{i}^{*}-\sum_{i \geq 1} \alpha_{n} \alpha_{i} x_{i}^{*} \\
& =\sum_{i>n} \alpha_{n} \alpha_{i} x_{i}^{*}-\sum_{i<n} \alpha_{n} \alpha_{i} x_{i}^{*} .
\end{aligned}
$$

Then by Theorem 3.6, (27) is well defined.

(ii): Apply (i) and Theorem 3.6.(i) directly.

(iii): Combine Theorem 3.6.(iii) \& (vi). 
[iv) Apply Theorem 3.6](v)] directly.

(v) Apply Theorem 3.6(viii) $\&$ (ix),

(vi) Since $\alpha \neq 0$, there exists $i_{0} \in \mathbb{N}$ such that $\alpha_{i_{0}} \neq 0$. Let $e_{i_{0}}:=(0, \ldots, 0,1,0, \ldots)$, i.e., the $i_{0}$ th is 1 and the others are 0 . Then by (29), we have

$$
S_{\alpha} e_{i_{0}}=\alpha_{i_{0}}\left(\alpha_{1}, \ldots, \alpha_{i_{0}-1}, 0,-\alpha_{i_{0}+1},-\alpha_{i_{0}+2}, \ldots\right) .
$$

Then

$$
\begin{aligned}
A^{*} e_{i_{0}} & =P_{\alpha} e_{i_{0}}-S_{\alpha} e_{i_{0}} \\
& =\alpha_{i_{0}}\left(0, \ldots, 0, \alpha_{i_{0}}, 2 \alpha_{i_{0}+1}, 2 \alpha_{i_{0}+2}, \ldots\right) .
\end{aligned}
$$

Now set $v_{0}^{*}:=e_{i_{0}}$ and $v_{0}:=3\|\alpha\|_{*}^{2} e_{i_{0}}$. Thus by (31),

$$
\begin{aligned}
v_{0}-A^{*} v_{0}^{*} & =3\|\alpha\|_{*}^{2} e_{i_{0}}-A^{*} e_{i_{0}} \\
& =\left(0, \ldots, 0,3\|\alpha\|_{*}^{2}-\alpha_{i_{0}}^{2},-2 \alpha_{i_{0}} \alpha_{i_{0}+1},-2 \alpha_{i_{0}} \alpha_{i_{0}+2}, \ldots\right) .
\end{aligned}
$$

Let $f:=\|\cdot\|$ on $X=c_{0}$. Then $f^{*}=\iota_{B_{X^{*}}}$ by [42, Corollary 2.4.16]. We have

$$
\begin{aligned}
f^{*}\left(v_{0}^{*}\right)+f^{* *}\left(v_{0}-A^{*} e_{i_{0}}\right) & =\iota_{B_{X^{*}}}\left(e_{i_{0}}\right)+\left\|v_{0}-A^{*} e_{i_{0}}\right\|_{*} \\
& =\|3\| \alpha\left\|_{*} e_{i_{0}}-A^{*} e_{i_{0}}\right\|_{*} \\
& <3\|\alpha\|_{*}^{2} \quad(\text { by (32) }) \\
& =\left\langle v_{0}, v_{0}^{*}\right\rangle .
\end{aligned}
$$

Hence by Theorem 3.t(xii), $F_{T_{\alpha}} \square_{1}\left(\|\cdot\| \oplus \iota_{B_{X^{*}}}\right)$ is not a BC-function.

(vii). Let $f:=\|\cdot\|$ on $X$. Since $\operatorname{dom} f^{* *}=X^{* *}$. Then apply Theorem 3.4(xiv),

(viii), By $\frac{1}{\sqrt{2}}<\|\alpha\|_{*} \leq 1$, take $\left|\alpha_{i_{0}}\right|^{2}>\frac{1}{2}$. Let $e_{i_{0}}$ be defined as in the proof of (vi). Then take $v_{1}^{*}:=\frac{1}{2} e_{i_{0}}$ and $v_{1}:=\left(1+\frac{1}{2} \alpha_{i_{0}}^{2}\right) e_{i_{0}}$.

By (31), we have

$$
v_{1}-A^{*} v_{1}^{*}=\left(0, \ldots, 0,1,-\alpha_{i_{0}} \alpha_{i_{0}+1},-\alpha_{i_{0}} \alpha_{i_{0}+2}, \ldots\right) .
$$

Since $\left|\alpha_{i_{0}} \alpha_{j}\right| \leq\|\alpha\|_{*}^{2} \leq 1, \forall j \in \mathbb{N}$, then

$$
\left\|v_{1}-A^{*} v_{1}^{*}\right\|_{*} \leq 1
$$

Let $f:=\frac{1}{2}\|\cdot\|^{2}$ on $X=c_{0}$. Then $f^{*}=\frac{1}{2}\|\cdot\|_{1}^{2}$ and $f^{* *}=\frac{1}{2}\|\cdot\|_{*}^{2}$. We have

$$
f^{*}\left(v_{1}^{*}\right)+f^{* *}\left(v_{1}-A^{*} v_{1}^{*}\right)=\frac{1}{2}\left\|v_{1}^{*}\right\|_{1}^{2}+\frac{1}{2}\left\|v_{1}-A^{*} v_{1}^{*}\right\|_{*}^{2}
$$




$$
\begin{aligned}
& \leq \frac{1}{8}+\frac{1}{2} \quad(\text { by }(\underline{34})) \\
& <\frac{\alpha_{i_{0}}^{2}}{4}+\frac{1}{2} \quad\left(\text { since } \alpha_{i_{0}}^{2}>1 / 2\right) \\
& =\left\langle v_{1}^{*}, v_{1}\right\rangle .
\end{aligned}
$$

Hence by Theorem 3.6(xii), $F_{T_{\alpha}} \square_{1}\left(\frac{1}{2}\|\cdot\|^{2} \oplus \frac{1}{2}\|\cdot\|_{*}^{2}\right)$ is not a BC-function.

(ix). Let $\lambda>0$ and $f:=\frac{\lambda}{2}\|\cdot\|^{2}$ on $X=c_{0}$. Then $f^{* *}=\frac{\lambda}{2}\|\cdot\|_{*}^{2}$. Then apply Theorem 3.6. (xiv).

(x). Since $c_{0}$ is separable by [26, Example 1.12.6] or [15, Proposition 1.26(ii)], by Fact 2.2, there exists a linear operator $L: c_{0} \rightarrow C[0,1]$ that is an isometry from $c_{0}$ to a subspace of $C[0,1]$. Then combine (vii) $\&$ (ix) and Corollary 2.18.

(xi) Combine (iii) (or (vii) or (ix) and Corollary 2.18.

(xii): To obtain the result on $T_{e}$, directly apply [iii)] (or see [2, Example 5.2]).

(xiii) Now $-G$ is type (D) but $G$ is not [2]. To see that $G$ is unique, note that $-G^{*}$ is monotone by Fact 2.14 and so provides the unique maximal extension. Since $G$ is skew and continuous, clearly, $-G^{*} x^{*}=G x^{*}, \forall x^{*} \in \ell^{1}$. Then Lemma 3.2 implies that $G$ is of type (BR). The uniqueness of $G$ was also verified in [1, Example 14.2.2].

Remark 4.2 The maximal monotonicity of the operator $T_{e}$ in Example 4.1](xii) was also verified by Voisei and Zălinescu in [39, Example 19] and later a direct proof given by Bueno and Svaiter in [12, Lemma 2.1]. Herein we have given a more concise proof of above results.

Bueno and Svaiter also showed that $T_{e}$ is not of type (D) in [12]. They also showed that each Banach space that contains an isometric (isomorphic) copy of $c_{0}$ is not of type (D) in [12]. Example 4.1](xi) recaptures their result, while Example 4.1](vi) \& (viii) provide a negative answer to Simons' [35, Problem 22.12].

Remark 4.3 (The continuous case) We recall that a Banach space $X$ is a conjugate monotone space if every continuous linear monotone operator on $X$ has a monotone conjugate. In particular this holds if every continuous linear monotone operator on $X$ is weakly compact. In consequence, a Banach lattice $X$ contains a complemented copy of $\ell^{1}$ if and only if it admits a non (D) continuous linear monotone operator, on using Fact 2.14 along with [2, Remark 5.5] and [2, Examples. 5.2 and 5.3].

Thus, in lattices such as $c_{0}, c$ and $C[0,1]$ only discontinuous linear monotone operators can fail to be of type (D). This subtlety escaped the current authors for fifteen years.

We now turn to a broader class of spaces: 


\subsection{Applications to more general nonreflexive spaces}

Our results below are facilitated by making use of Schauder basis structure [16].

Definition 4.4 We say $\left(e_{n}, e_{n}^{*}\right)_{n \in \mathbb{N}}$ in $X \times X^{*}$ is a Schauder basis of $X$ if for every $x \in X$ there exists a unique sequence $\left(\alpha_{n}\right)_{n \in \mathbb{N}}$ in $\mathbb{R}$ such that $x=\sum_{n>1} \alpha_{n} e_{n}$, where $\alpha_{n}=\left\langle x, e_{n}^{*}\right\rangle$ and $\left\langle e_{i}, e_{j}^{*}\right\rangle=\delta_{i, j}, \forall i, j \in \mathbb{N}$.

Definition 4.5 Let $\left(e_{n}, e_{n}^{*}\right)_{n \in \mathbb{N}}$ in $X \times X^{*}$ be a Schauder basis of $X$. We say the basis is shrinking if $\overline{\operatorname{span}\left\{e_{n}^{*} \mid n \in \mathbb{N}\right\}}=X^{*}$.

In particular, a Banach space with a shrinking basis has a separable dual and so is an Asplund space [16].

Fact 4.6 (See [16, Lemma 4.7(iii) and Facts 4.11(ii)\&(iii)] or [15, Lemma 6.2(iii) and Facts 6.6(ii)\&(iii)] .) Let $\left(e_{n}, e_{n}^{*}\right)_{n \in \mathbb{N}}$ in $X \times X^{*}$ be a Schauder basis of $X$. Then

(i) $\lim _{n} \sum_{i=1}^{n}\left\langle x, e_{i}^{*}\right\rangle e_{i}=x, \quad \forall x \in X$;

(ii) $\sum_{i=1}^{n}\left\langle x^{*}, e_{i}\right\rangle e_{i}^{*}$ weak $k^{*}$ converges to $x^{*}$, written as, $\sum_{i=1}^{n}\left\langle x^{*}, e_{i}\right\rangle e_{i}^{*} \stackrel{\mathrm{w}^{*}}{\rightarrow} x^{*}, \quad \forall x^{*} \in X^{*}$;

(iii) $\left(e_{n}^{*}, e_{n}\right)_{n \in \mathbb{N}}$ in $X^{*} \times X^{* *}$ is a Schauder basis of $\overline{\operatorname{span}\left\{e_{n}^{*} \mid n \in \mathbb{N}\right\}}$.

Lemma 4.7 Let $\left(e_{n}, e_{n}^{*}\right)_{n \in \mathbb{N}}$ in $X \times X^{*}$ be a Schauder basis of $X$. Then $e_{n}^{*} \stackrel{\mathrm{w}^{*}}{\rightarrow} 0$ whenever $\liminf _{n \in \mathbb{N}}\left\|e_{n}\right\|>0$.

Proof. Let $x \in X$. Since $\left\|\left\langle x, e_{n}^{*}\right\rangle e_{n}\right\| \rightarrow 0$ because of Fact 4.6](i), and since $\liminf \inf _{n \in \mathbb{N}}\left\|e_{n}\right\|>0$, we have $\left\langle x, e_{n}^{*}\right\rangle \rightarrow 0$. Hence $e_{n}^{*} \stackrel{\mathrm{w}^{*}}{\rightarrow} 0$ as $n \rightarrow \infty$.

The proof of Example 4.8(i) was inspired by that [3, Proposition 3.5].

Example 4.8 (Schauder basis) Let $\left(e_{n}, e_{n}^{*}\right)_{n \in \mathbb{N}}$ in $X \times X^{*}$ be a Schauder basis of $X$. Assume that for some $e \in X^{* *}$ we have

$$
\sum_{i=1}^{n} e_{i} \stackrel{\mathrm{w}^{*}}{\rightarrow} e \in X^{* *}
$$

Let $A: X \rightrightarrows X^{*}$ be defined by

$$
\operatorname{gra} A:=\left\{\left(\sum_{n}\left(-\sum_{i>n}\left\langle e_{i}, y^{*}\right\rangle+\sum_{i<n}\left\langle e_{i}, y^{*}\right\rangle\right) e_{n}, y^{*}\right) \in X \times X^{*} \mid y^{*} \in\{e\}_{\perp}\right\} .
$$

Assume that $\lim \inf \left\|e_{n}\right\|>0$. Then the following hold. 
(i) $A$ is a maximally monotone and linear skew operator.

(ii) $A$ is not of type (BR).

(iii) $A$ is not of type (D).

(iv) $A$ is not unique.

(v) Every Banach space containing a copy of $X$ is not of type (D).

Proof. (i): First, we show $A$ is skew. Let $\left(y, y^{*}\right) \in \operatorname{gra} A$. Then $\left\langle e, y^{*}\right\rangle=0$ and $y=\sum_{n=1}^{\infty}\left(-\sum_{i>n}\left\langle e_{i}, y^{*}\right\rangle+\sum_{i<n}\left\langle e_{i}, y^{*}\right\rangle\right) e_{n}$. By the assumption that $\sum_{i=1}^{n} e_{i} \stackrel{\mathrm{w}^{*}}{\rightarrow} e \in X^{* *}$, we have

$$
s:=\sum_{i \geq 1}\left\langle e_{i}, y^{*}\right\rangle=\left\langle e, y^{*}\right\rangle=0
$$

Thus,

$$
\begin{aligned}
\left\langle y, y^{*}\right\rangle= & \left\langle\sum_{n}\left(-\sum_{i>n}\left\langle e_{i}, y^{*}\right\rangle+\sum_{i<n}\left\langle e_{i}, y^{*}\right\rangle\right) e_{n}, y^{*}\right\rangle \\
= & \lim _{k}\left\langle\sum_{n=1}^{k}\left(-\sum_{i>n}\left\langle e_{i}, y^{*}\right\rangle+\sum_{i<n}\left\langle e_{i}, y^{*}\right\rangle\right) e_{n}, y^{*}\right\rangle \quad \text { (by Fact 4.6](i)] } \\
= & \lim _{k} \sum_{n=1}^{k}\left(-\sum_{i>n}\left\langle e_{i}, y^{*}\right\rangle+\sum_{i<n}\left\langle e_{i}, y^{*}\right\rangle\right)\left\langle e_{n}, y^{*}\right\rangle \\
= & -\lim _{k} \sum_{n=1}^{k}\left(\sum_{i>n}\left\langle e_{i}, y^{*}\right\rangle-\sum_{i<n}\left\langle e_{i}, y^{*}\right\rangle\right)\left\langle e_{n}, y^{*}\right\rangle \\
= & -\lim _{k} \sum_{n=1}^{k}\left(\sum_{i \geq n+1}\left\langle e_{i}, y^{*}\right\rangle+\sum_{i \geq n}\left\langle e_{i}, y^{*}\right\rangle\right)\left\langle e_{n}, y^{*}\right\rangle \quad(\text { by (36) }) \\
= & -\lim _{k}\left(\left\langle e_{1}, y^{*}\right\rangle \sum_{i \geq 1}\left\langle e_{i}, y^{*}\right\rangle+\left\langle e_{2}, y^{*}\right\rangle \sum_{i \geq 2}\left\langle e_{i}, y^{*}\right\rangle+\cdots+\left\langle e_{k}, y^{*}\right\rangle \sum_{i \geq k}\left\langle e_{i}, y^{*}\right\rangle\right. \\
& \left.+\left\langle e_{1}, y^{*}\right\rangle \sum_{i \geq 2}\left\langle e_{i}, y^{*}\right\rangle+\left\langle e_{2}, y^{*}\right\rangle \sum_{i \geq 3}\left\langle e_{i}, y^{*}\right\rangle+\cdots+\left\langle e_{k}, y^{*}\right\rangle \sum_{i \geq k+1}\left\langle e_{i}, y^{*}\right\rangle\right) \\
= & -\lim _{k}\left(s\left\langle e_{1}, y^{*}\right\rangle+\left(s-\left\langle e_{1}, y^{*}\right\rangle\right)\left\langle e_{2}, y^{*}\right\rangle+\cdots+\left(s-\sum_{i=1}^{k-1}\left\langle e_{i}, y^{*}\right\rangle\right)\left\langle e_{k}, y^{*}\right\rangle\right. \\
& \left.+\left(s-\left\langle e_{1}, y^{*}\right\rangle\right)\left\langle e_{1}, y^{*}\right\rangle+\left(s-\sum_{i=1}^{2}\left\langle e_{i}, y^{*}\right\rangle\right)\left\langle e_{2}, y^{*}\right\rangle+\cdots+\left(s-\sum_{i=1}^{k}\left\langle e_{i}, y^{*}\right\rangle\right)\left\langle e_{k}, y^{*}\right\rangle\right)
\end{aligned}
$$




$$
\begin{aligned}
= & -\lim _{k}\left(s \sum_{i=1}^{k}\left\langle e_{i}, y^{*}\right\rangle-\left\langle e_{1}, y^{*}\right\rangle\left\langle e_{2}, y^{*}\right\rangle-\sum_{i=1}^{2}\left\langle e_{i}, y^{*}\right\rangle\left\langle e_{3}, y^{*}\right\rangle-\cdots-\sum_{i=1}^{k-1}\left\langle e_{i}, y^{*}\right\rangle\left\langle e_{k}, y^{*}\right\rangle\right. \\
& \left.+s \sum_{i=1}^{k}\left\langle e_{i}, y^{*}\right\rangle-\sum_{i=1}^{k}\left\langle e_{i}, y^{*}\right\rangle^{2}-\left\langle e_{1}, y^{*}\right\rangle\left\langle e_{2}, y^{*}\right\rangle-\cdots-\sum_{i=1}^{k-1}\left\langle e_{i}, y^{*}\right\rangle\left\langle e_{k}, y^{*}\right\rangle\right) \\
= & -\lim _{k}\left[2 s \sum_{i=1}^{k}\left\langle e_{i}, y^{*}\right\rangle-\left(\sum_{i=1}^{k}\left\langle e_{i}, y^{*}\right\rangle\right)^{2}\right] \\
= & \left.\left.-\left(2 s^{2}-s^{2}\right)=-s^{2}=0 . \quad \text { (by (136) }\right)\right)
\end{aligned}
$$

Hence $A$ is skew.

To show maximality, let $\left(x, x^{*}\right) \in X \times X^{*}$ be monotonically related to gra $A$. By Fact 2.6 , we have

$$
\left\langle y^{*}, x\right\rangle+\left\langle x^{*}, y\right\rangle=0, \quad \forall\left(y, y^{*}\right) \in \operatorname{gra} A
$$

By (35), we have

$$
\left\langle e, e_{n}^{*}\right\rangle=\sum_{i \geq 1}\left\langle e_{i}, e_{n}^{*}\right\rangle=\delta_{n, n}=1, \quad \forall n \in \mathbb{N}
$$

Let $y^{*}:=-e_{1}^{*}+e_{n}^{*}(n \geq 2)$ and $y:=-e_{1}-2 \sum_{i=2}^{n-1} e_{i}-e_{n}$. By (39), we have $\left\langle e, y^{*}\right\rangle=0$. Hence $y^{*} \in\{e\}_{\perp}$ and $\left(y, y^{*}\right) \in \operatorname{gra} A$. Using (38),

$$
-\left\langle x, e_{1}^{*}\right\rangle+\left\langle x, e_{n}^{*}\right\rangle-\left\langle x^{*}, e_{1}\right\rangle-\left\langle x^{*}, e_{n}\right\rangle-2 \sum_{i=2}^{n-1}\left\langle x^{*}, e_{i}\right\rangle=0 .
$$

Thus, we have

$$
\left\langle x, e_{n}^{*}\right\rangle=\left\langle x, e_{1}^{*}\right\rangle-\left\langle x^{*}, e_{1}\right\rangle+\left\langle x^{*}, e_{n}\right\rangle+2 \sum_{i=1}^{n-1}\left\langle x^{*}, e_{i}\right\rangle .
$$

As $\sum_{i \geq 1}\left\langle e_{i}, z^{*}\right\rangle=\left\langle e, z^{*}\right\rangle\left(\forall z^{*} \in X^{*}\right)$, we have $\left\langle x^{*}, e_{n}\right\rangle \rightarrow 0$.

Hence, by Lemma 4.7 - since $\lim \inf \left\|e_{n}\right\|>0$ - and (40),

$$
-2 \sum_{i \geq 1}\left\langle x^{*}, e_{i}\right\rangle=\left\langle x, e_{1}^{*}\right\rangle-\left\langle x^{*}, e_{1}\right\rangle .
$$

Next we show $-2 \sum_{i \geq 1}\left\langle x^{*}, e_{i}\right\rangle=\left\langle x, e_{1}^{*}\right\rangle-\left\langle x^{*}, e_{1}\right\rangle=0$. Let $t=\sum_{i \geq 1}\left\langle x^{*}, e_{i}\right\rangle$. Then by (40) and (41),

$$
x=\sum_{n \geq 1}\left\langle x, e_{n}^{*}\right\rangle e_{n}
$$




$$
\begin{aligned}
& =\sum_{n \geq 1}\left(-2 \sum_{i \geq 1}\left\langle x^{*}, e_{i}\right\rangle+2 \sum_{i<n}\left\langle x^{*}, e_{i}\right\rangle+\left\langle x^{*}, e_{n}\right\rangle\right) e_{n} \\
& =\sum_{n \geq 1}\left(-2 \sum_{i \geq n}\left\langle x^{*}, e_{i}\right\rangle+\left\langle x^{*}, e_{n}\right\rangle\right) e_{n} \\
& =\sum_{n \geq 1}\left(-\sum_{i \geq n}\left\langle x^{*}, e_{i}\right\rangle-\sum_{i \geq n}\left\langle x^{*}, e_{i}\right\rangle+\left\langle x^{*}, e_{n}\right\rangle\right) e_{n} \\
& =\sum_{n \geq 1}\left(-\sum_{i \geq n}\left\langle x^{*}, e_{i}\right\rangle-\sum_{i \geq n+1}\left\langle x^{*}, e_{i}\right\rangle\right) e_{n} .
\end{aligned}
$$

Using $(0,0) \in \operatorname{gra} A$, as in the proof of (37), shows

$$
\begin{aligned}
0 \geq-\left\langle x^{*}, x\right\rangle & =\left\langle\sum_{n \geq 1}\left(\sum_{i \geq n}\left\langle x^{*}, e_{i}\right\rangle+\sum_{i \geq n+1}\left\langle x^{*}, e_{i}\right\rangle\right) e_{n}, x^{*}\right\rangle \\
& =\lim _{k}\left\langle\sum_{n=1}^{k}\left(\sum_{i \geq n}\left\langle x^{*}, e_{i}\right\rangle+\sum_{i \geq n+1}\left\langle x^{*}, e_{i}\right\rangle\right) e_{n}, x^{*}\right\rangle \\
& =2 t^{2}-t^{2}=t^{2} .
\end{aligned}
$$

Hence $t=0$. By (42),

$$
x=\sum_{n \geq 1}\left(-\sum_{i>n}\left\langle x^{*}, e_{i}\right\rangle+\sum_{i<n}\left\langle x^{*}, e_{i}\right\rangle\right) e_{n} .
$$

Hence $\left(x, x^{*}\right) \in \operatorname{gra} A$. Thus, $A$ is maximally monotone.

(ii): Suppose to the contrary that $A$ is of type (BR). One checks that $\left(e_{1}, e_{1}^{*}\right) \in$ gra $A^{*}$ and $\left\langle e, e_{1}^{*}\right\rangle=\lim _{n}\left\langle\sum_{i=1}^{n} e_{i}, e_{1}^{*}\right\rangle=1$. Thus, $\left(e_{1},-e_{1}^{*}\right) \in \operatorname{gra}\left(-A^{*}\right) \cap X \times X^{*}$ and $-e_{1}^{*} \notin\{e\}_{\perp}$. Since $\overline{\operatorname{ran} A} \subseteq\{e\}_{\perp},-e_{1}^{*} \notin \overline{\operatorname{ran} A}$. Then $\left.\inf _{\left(a, a^{*}\right) \in \operatorname{gra} A}\left\langle e_{1}-a,-e_{1}^{*}-a^{*}\right\rangle=\left\langle e_{1},-e_{1}^{*}\right\rangle=-1\right\rangle-\infty$. Then by Fact $2.10,-e_{1}^{*} \in \overline{\operatorname{ran} A}$, which contradicts that $-e_{1}^{*} \notin \overline{\operatorname{ran} A}$. Hence $A$ is not of type (BR).

(iii): By Fact 2.11 and (ii), $A$ is not of type (NI) and hence $A$ is not of type (D) by Fact 2.7. Alternative Proof: Clearly, $(e, 0) \in \operatorname{gra} A^{*}$ and thus $e \in \operatorname{ker} A^{*}$. By the proof of (ii), $\left(e_{1}, e_{1}^{*}\right) \in \operatorname{gra} A^{*}$ and $\left\langle e, e_{1}^{*}\right\rangle=1$. Hence $e \notin\left(\operatorname{ran} A^{*}\right)^{\perp}$. Hence $A^{*}$ is not monotone by Lemma 2.13, Then Fact 2.14 shows $A$ is not of type (D).

(iv): Apply (iii)\&(ii) and Corollary 3.3 directly.

(v): Combine (i)\&(iii) and Corollary 2.18.

We shall especially exploit the lovely properties of the James space: 
Definition 4.9 The James space, $\mathbf{J}$, consists of all the sequences $x=\left(x_{n}\right)_{n \in \mathbb{N}}$ in $c_{0}$ with the finite norm

$$
\|x\|:=\sup _{n_{1}<\cdots<n_{k}}\left(\left(x_{n_{1}}-x_{n_{2}}\right)^{2}+\left(x_{n_{2}}-x_{n_{3}}\right)^{2}+\cdots+\left(x_{n_{k-1}}-x_{n_{k}}\right)^{2}\right)^{\frac{1}{2}} .
$$

Fact 4.10 (See [16, page 205] or [15, Claim, page 185].) The space $\mathbf{J}$ is constructed to be of codimension-one in $\mathbf{J}^{* *}$. Indeed, $\mathbf{J}^{* *}=\mathbf{J} \oplus \operatorname{span}\{e\}$ where $e:=(1,1, \ldots, 1, \ldots)$ is the constant sequence in $c(\mathbb{N}) \subset \ell^{\infty}$. Thus, $\mathbf{J}$ is a separable Asplund space, equivalently $\mathbf{J}^{*}$ is separable [11, 16, 15], and non-reflexive. Inter alia, the basis $\left(e_{n}, e_{n}^{*}\right)_{n \in \mathbb{N}}$ is a shrinking Schauder basis in $\mathbf{J}$ and $\left(e_{n}^{*}, e_{n}\right)_{n \in \mathbb{N}}$ is a basis for $\mathbf{J}^{*}$, where $e_{n}=(0, \ldots, 0,1,0, \ldots)$, i.e., the $n$th is 1 and the others are 0 .

Corollary 4.11 (James space) Let $X$ be the James space, J. Let $e_{n}$ be defined as in Fact 4.10, and let $A$ be defined as in Example 4.8. Then $A$ is a maximally monotone and skew operator that is neither of type (BR) nor unique and so $A$ is not of type (D). Hence, every Banach space that contains an isomorphic copy of $\boldsymbol{J}$ is not of type (D).

Proof. To apply Example 4.8 we need only verify that (35) holds. To see this is so, we note that $\left(\sum_{i=1}^{n} e_{i}\right)_{n \in \mathbb{N}}$ lies in $B_{\mathbf{J}^{* *}}$ - directly from the definition of the norm in $\mathbf{J}$. Now by the Banach-Alaoglu theorem and [16, Proposition 3.103, page 128] or [15, Proposition 3.24, page 72$]$, we have the vector $e=(1,1, \ldots, 1, \ldots)$ is the unique $w^{*}$ limit of $\left(\sum_{i=1}^{n} e_{i}\right)_{n \in \mathbb{N}^{*}}$.

An easier version of the same argument leads to a recovery of part of Example 4.1;

Corollary $4.12\left(c_{0}\right)$ Let $X=c_{0}$. Let $e_{n}$ be defined as in Fact 4.10 and $e:=(1,1, \ldots, 1, \ldots)$. Let $A$ be defined as in Example 4.8 (thus $A=T_{e}$ in Example 4.1](xii)). Then $A$ is a maximally monotone and skew operator that is neither of type (BR) nor unique and so $A$ is not of type (D). Hence, every Banach space that contains an isomorphic copy of $c_{0}$ is not of type (D).

We finish our set of core examples by dealing with the dual space $\mathbf{J}^{*}$.

Example 4.13 (Shrinking Schauder basis) Let $\left(e_{n}, e_{n}^{*}\right)_{n \in \mathbb{N}}$ in $X \times X^{*}$ be a shrinking Schauder basis of $X$. Assume that $\sum_{i=1}^{n} e_{i} \stackrel{\mathrm{w}^{*}}{\rightarrow} e$ for some $e \in X^{* *}$. Let $A: X^{*} \rightrightarrows X^{* *}$ be defined by

$$
\operatorname{gra} A=\left\{\left(y^{*}, y^{* *}\right) \in X^{*} \times X^{* *} \mid \sum_{n=1}^{k}\left(\sum_{i>n}\left\langle e_{i}, y^{*}\right\rangle-\sum_{i<n}\left\langle e_{i}, y^{*}\right\rangle\right) e_{n} \stackrel{\mathrm{w}^{*}}{\rightarrow} y^{* *}\right\}
$$

Then $A$ is a maximally monotone and linear skew operator, which is of type (BR).

In particular, let $\left(e_{n}\right)_{n \in \mathbb{N}}$ and $e$ be defined as in Fact 4.10. Then $A+\langle\cdot, e\rangle e$ is a maximally monotone operator that is neither of type (D) nor unique; and every Banach space containing a copy of $\mathbf{J}^{*}$ is not of type (D). 
Proof. Again, we first show $A$ is skew. Let $\left(y^{*}, y^{* *}\right) \in \operatorname{gra} A$. Then

$$
\sum_{n=1}^{k}\left(\sum_{i>n}\left\langle e_{i}, y^{*}\right\rangle-\sum_{i<n}\left\langle e_{i}, y^{*}\right\rangle\right) e_{n} \stackrel{\mathrm{w}^{*}}{\rightarrow} y^{* *}
$$

By the assumption that $\sum_{i=1}^{n} e_{i} \stackrel{\mathrm{w}^{*}}{\rightarrow} e \in X^{* *}$, we have

$$
s:=\sum_{i \geq 1}\left\langle e_{i}, y^{*}\right\rangle=\left\langle e, y^{*}\right\rangle
$$

Thus,

$$
\begin{aligned}
\left\langle y^{* *}, y^{*}\right\rangle & =\lim _{k}\left\langle\sum_{n=1}^{k}\left(\sum_{i>n}\left\langle e_{i}, y^{*}\right\rangle-\sum_{i<n}\left\langle e_{i}, y^{*}\right\rangle\right) e_{n}, y^{*}\right\rangle \\
& =\lim _{k} \sum_{n=1}^{k}\left(\sum_{i>n}\left\langle e_{i}, y^{*}\right\rangle-\sum_{i<n}\left\langle e_{i}, y^{*}\right\rangle\right)\left\langle e_{n}, y^{*}\right\rangle \\
& \left.=\lim _{k} \sum_{n=1}^{k}\left(\sum_{i \geq n+1}\left\langle e_{i}, y^{*}\right\rangle+\sum_{i \geq n}\left\langle e_{i}, y^{*}\right\rangle-s\right)\left\langle e_{n}, y^{*}\right\rangle \quad \text { (by (444) }\right) \\
& =-s \lim _{k} \sum_{n=1}^{k}\left\langle e_{n}, y^{*}\right\rangle+\lim _{k} \sum_{n=1}^{k}\left(\sum_{i \geq n+1}\left\langle e_{i}, y^{*}\right\rangle+\sum_{i \geq n}\left\langle e_{i}, y^{*}\right\rangle\right)\left\langle e_{n}, y^{*}\right\rangle \\
& =-s^{2}+\left(2 s^{2}-s^{2}\right)=0 \quad(\text { as in the proof of (37) }) .
\end{aligned}
$$

Hence $A$ is skew.

Now we confirm maximality. Let $\left(x^{*}, x^{* *}\right) \in X^{*} \times X^{* *}$ be monotonically related to gra $A$. By Fact 2.6, we have

$$
\left\langle y^{*}, x^{* *}\right\rangle+\left\langle x^{*}, y^{* *}\right\rangle=0, \quad \forall\left(y^{*}, y^{* *}\right) \in \operatorname{gra} A .
$$

Fix $n \in \mathbb{N}$ and set $y^{*}:=e_{n}^{*}$. Then $\sum_{j=1}^{k}\left(\sum_{i>j}\left\langle e_{i}, y^{*}\right\rangle-\sum_{i<j}\left\langle e_{i}, y^{*}\right\rangle\right) e_{j}=\sum_{j=1}^{n-1} e_{j}-$ $\sum_{j=n+1}^{k} e_{j}$. By the assumption that $\sum_{i=1}^{k} e_{i} \stackrel{\mathrm{w}^{*}}{\rightarrow} e$, we have

$$
\sum_{j=1}^{n-1} e_{j}-\sum_{j=n+1}^{k} e_{j} \stackrel{\mathrm{w}^{*}}{\longrightarrow} 2 \sum_{j=1}^{n-1} e_{j}+e_{n}-e .
$$

Hence $\left(e_{n}^{*}, 2 \sum_{j=1}^{n-1} e_{j}+e_{n}-e\right) \in \operatorname{gra} A$. Then by (45),

$$
\left\langle x^{* *}, e_{n}^{*}\right\rangle+2 \sum_{j=1}^{n-1}\left\langle x^{*}, e_{j}\right\rangle+\left\langle x^{*}, e_{n}\right\rangle-\left\langle x^{*}, e\right\rangle=0 .
$$


Since $\sum_{j \geq 1}\left\langle x^{*}, e_{j}\right\rangle=\left\langle x^{*}, e\right\rangle$, we have

$$
\left\langle x^{* *}, e_{n}^{*}\right\rangle=-2 \sum_{j=1}^{n-1}\left\langle x^{*}, e_{j}\right\rangle-\left\langle x^{*}, e_{n}\right\rangle+\left\langle x^{*}, e\right\rangle=\sum_{j>n}\left\langle x^{*}, e_{j}\right\rangle-\sum_{j<n}\left\langle x^{*}, e_{j}\right\rangle .
$$

By Fact 4.6](ii)] [(iii), $\sum_{n=1}^{k}\left(\sum_{j>n}\left\langle x^{*}, e_{j}\right\rangle-\sum_{j<n}\left\langle x^{*}, e_{j}\right\rangle\right) e_{n} \stackrel{\mathrm{w}^{*}}{\rightarrow} x^{* *}$. Hence $\left(x^{*}, x^{* *}\right) \in \operatorname{gra} A$. Thus, $A$ is maximally monotone.

We next show that $A$ is of type (BR). Let $\left(z^{*}, z^{* *}\right) \in \operatorname{gra}\left(-A^{*}\right) \cap X^{*} \times X^{* *}$. Much as in the proof above starting at (45), we have $\left(z^{*}, z^{* *}\right) \in \operatorname{gra} A$. Thus, gra $\left(-A^{*}\right) \cap X \times X^{* *} \subseteq \operatorname{gra} A$. Then by Lemma 3.2, $A$ is of type (BR).

We turn to the particularization. By Fact 4.10, $\left(e_{n}, e_{n}^{*}\right)_{n \in \mathbb{N}}$ is a shrinking Schauder basis for J. By Fact 2.15 since $A$ is maximal, $T=A+\langle\cdot, e\rangle e=A+\partial \frac{1}{2}\langle\cdot, e\rangle^{2}$ is maximally monotone. Since $A$ is skew, we have

$$
\left\langle x^{*}, x^{* *}\right\rangle=\left\langle x^{*}, e\right\rangle^{2}, \quad \forall\left(x^{*}, x^{* *}\right) \in \operatorname{gra} T .
$$

Now we claim that

$$
e \notin \overline{\operatorname{ran} T}
$$

Let $\left(y^{*}, y^{* *}\right)$ in gra $T$. Then

$$
\begin{aligned}
& \sum_{j=1}^{k}\left(2 \sum_{i>j}\left\langle e_{i}, y^{*}\right\rangle+\left\langle e_{j}, y^{*}\right\rangle\right) e_{j} \\
& =\sum_{j=1}^{k}\left(\left\langle y^{*}, e\right\rangle+\sum_{i>j}\left\langle e_{i}, y^{*}\right\rangle-\sum_{i<j}\left\langle e_{i}, y^{*}\right\rangle\right) e_{j} \quad\left(\text { by } \sum_{i \geq 1}\left\langle e_{j}, y^{*}\right\rangle=\left\langle e, y^{*}\right\rangle\right) \\
& =\left\langle y^{*}, e\right\rangle \sum_{j=1}^{k} e_{j}+\sum_{j=1}^{k}\left(\sum_{i>j}\left\langle e_{i}, y^{*}\right\rangle-\sum_{i<j}\left\langle e_{i}, y^{*}\right\rangle\right) e_{j} \stackrel{\mathrm{w}^{*}}{\rightarrow} y^{* *} .
\end{aligned}
$$

Then by (49),

$$
\begin{aligned}
\lim _{k}\left\langle y^{* *}, e_{k}^{*}\right\rangle & =\lim _{k} \lim _{L}\left\langle\sum_{j=1}^{L}\left(2 \sum_{i>j}\left\langle e_{i}, y^{*}\right\rangle+\left\langle e_{j}, y^{*}\right\rangle\right) e_{j}, e_{k}^{*}\right\rangle \\
& =\lim _{k}\left(2 \sum_{i>k}\left\langle e_{i}, y^{*}\right\rangle+\left\langle e_{k}, y^{*}\right\rangle\right) \\
& =0 \quad\left(\text { by } \sum_{k \geq 1}\left\langle e_{k}, y^{*}\right\rangle=\left\langle e, y^{*}\right\rangle\right) .
\end{aligned}
$$


Then by Fact 4.10, $y^{* *} \in \mathbf{J}$ and hence $\operatorname{ran} T \subseteq \mathbf{J}$. Thus

$$
\overline{\operatorname{ran} T} \subseteq \mathbf{J} .
$$

Since $\left\langle e, e_{k}^{*}\right\rangle=1, \forall k \in \mathbb{N}$, then by Lemma 4.7, $e \notin \mathbf{J}$. Then by (51), we have (48) holds. Combining (47), (48) and Proposition 3.5, $T=A+\langle\cdot, e\rangle e$ is neither of type (D) nor unique.

This suffices to finish the argument.

Remark 4.14 $\left(\ell^{1}\right)$ A simpler version of the previous result recovers the original result that $\ell^{1}$ admits Gossez type operators.

\section{Conclusion}

We have provided various tools for the further construction of pathological maximally monotone operators and related Fitzpatrick functions. In particular, we have shown - building on the work of Gossez, Phelps, Simons, Svaiter, Bueno and others, and our own previous work - that every Banach space which contains an isomorphic copy of either the James space $\mathbf{J}$ or its dual $\mathbf{J}^{*}$, or $c_{0}$ or its dual $\ell^{1}$, admits an operator which is not of type (D). We observe that the type (D) property is preserved by direct sums and subspaces. Since every separable space is isometric to a quotient space of $\ell^{1}$ [16, Theorem 5.1, page 237] or [15, Theorem 5.9, page 140], it is not preserved by quotients.

Example 5.1 (Summary) We list some of the salient spaces covered by our work:

(i) Separable Asplund spaces: both $\mathbf{J}$ and $c_{0}$ afford examples.

(ii) Separable spaces whose dual is nonseparable and contain $\ell^{1}$ : include $\mathrm{E}^{1}([0,1]), C([0,1])$ and its superspace $L^{\infty}([0,1])$.

(iii) Separable spaces whose dual is nonseparable but does not contain a copy of $\ell^{1}$ : these include the James tree space JT [16, page 233] or [15, page 199] as it contains many copies of $\mathbf{J}$ (and of $\ell^{2}(\mathbb{N})$ ).

One remaining potential type (D) space is Gowers' space [21] which is a non-reflexive Banach space containing neither $c_{0}, \ell^{1}$ or any reflexive subspace.

As we saw, the maximally monotone operators in our examples - with the exception of the Gossez operator - that are not of type (D) are actually not unique. This raises the question of how in generality to construct maximally monotone linear relations that are not of type (D) but that are unique. 


\subsection{Graphic of classes of maximally monotone operators}

We capture much of the current state of knowledge in the following diagram in which the notation below is used.

"*" refers to skew operators such as $T$ in Theorem 3.6, $T_{\alpha}$ in Example 4.1,

$A$ in Example 4.8, $A$ in Corollary 4.11, and $A$ in Corollary 4.12.

"**" refers to the operators such as $A \& T$ in Theorem 3.6, $A_{\alpha} \& T_{\alpha}$ in Example 4.1,

$A$ in Example 4.8, $A$ in Corollary 4.11, $A$ in Corollary 4.12,

and $A+\langle\cdot, e\rangle e$ in Example 4.13 .

“***" denotes maximally monotone and unique operators with non affine graphs.

We let (ANA), (FP) and (FPV) respectively denote the other monotone operator classes "almost negative alignment", "Fitzpatrick-Phelps" and "Fitzpatrick-Phelps-Veronas". Then by [35, 11, 9, 5, 33, 25, 36, 41], we have the following relationships.

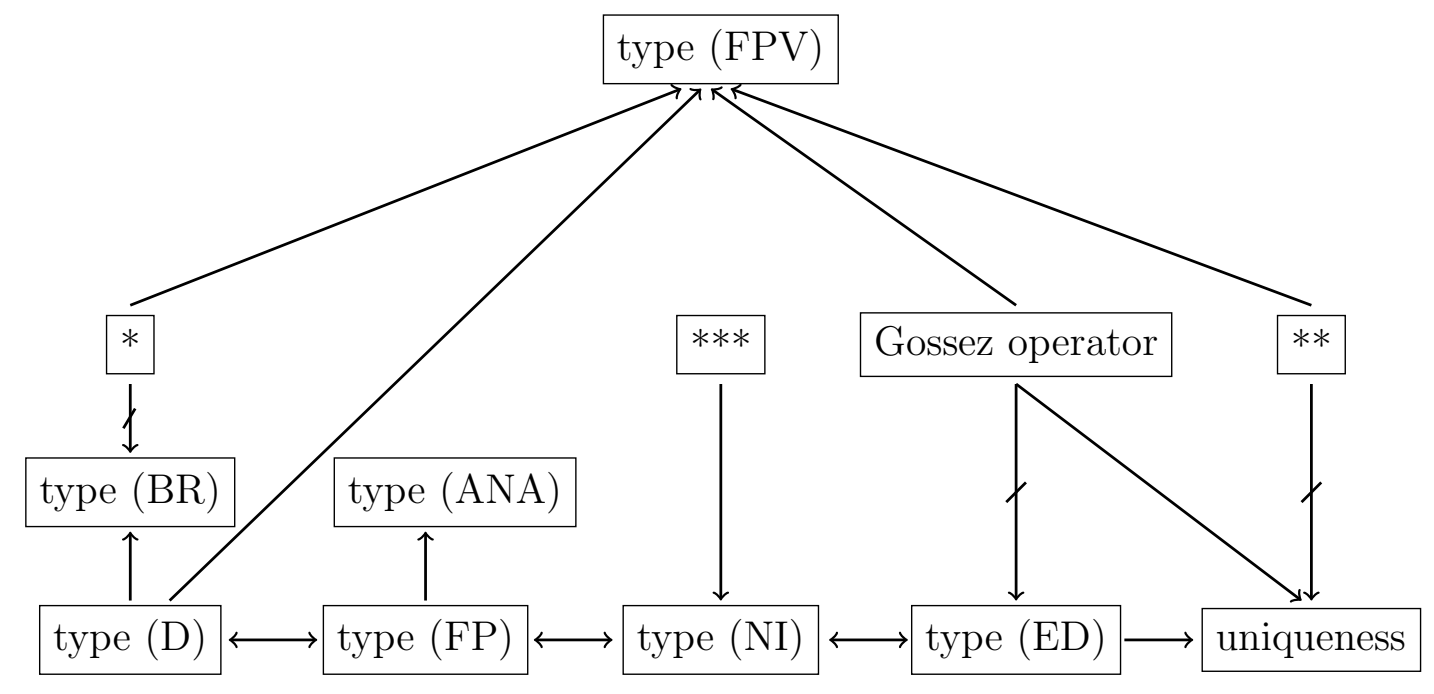

The following four questions are left open.

(i) Is every maximally monotone operator necessarily of type (FPV)?

(ii) Is every maximally monotone operator necessarily of type (ANA)?

(iii) Is every maximally monotone linear relation necessarily of type (ANA)?

(iv) Is every maximally monotone operator of type (BR) necessarily of type (ANA)?

The first of these is especially important, being closely related to the sum theorem in general Banach space (see [35, 11, 9, 40]). 
Acknowledgments. Heinz Bauschke was partially supported by the Natural Sciences and Engineering Research Council of Canada and by the Canada Research Chair Program. Jonathan Borwein was partially supported by the Australian Research Council. Xianfu Wang was partially supported by the Natural Sciences and Engineering Research Council of Canada. 


\section{References}

[1] H.H. Bauschke, Projection Algorithms and Monotone Operators, PhD thesis, Simon Fraser University, Department of Mathematics, Burnaby, British Columbia V5A 1S6, Canada, August 1996; Available at http://www.cecm.sfu.ca/preprints/1996pp.html.

[2] H.H. Bauschke and J.M. Borwein, "Maximal monotonicity of dense type, local maximal monotonicity, and monotonicity of the conjugate are all the same for continuousi linear operators", Pacific Journal of Mathematics, vol. 189, pp. 1-20, 1999.

[3] H.H. Bauschke, X. Wang, and L. Yao, "Examples of discontinuous maximal monotone linear operators and the solution to a recent problem posed by B.F. Svaiter", Journal of Mathematical Analysis and Applications, vol. 370, pp. 224-241, 2010.

[4] H.H. Bauschke, J.M. Borwein, X. Wang and L. Yao, "For maximally monotone linear relations, dense type, negative-infimum type, and Fitzpatrick-Phelps type all coincide with monotonicity of the adjoint", submitted; http://arxiv.org/abs/1103.6239v1, March 2011.

[5] H.H. Bauschke, J.M. Borwein, X. Wang, and L. Yao, "Every maximally monotone operator of Fitzpatrick-Phelps type is actually of dense type", submitted; http://arxiv.org/abs/1104.0750v1, April 2011.

[6] H.H. Bauschke and P.L. Combettes, Convex Analysis and Monotone Operator Theory in Hilbert Spaces, Springer-Verlag, 2011.

[7] J.M. Borwein, "Maximal monotonicity via convex analysis", Journal of Convex Analysis, vol. 13, pp. 561-586, 2006.

[8] J.M. Borwein, "Maximality of sums of two maximal monotone operators in general Banach space", Proceedings of the American Mathematical Society, vol. 135, pp. 39173924, 2007.

[9] J.M. Borwein, "Fifty years of maximal monotonicity", Optimization Letters, vol. 4, pp. 473-490, 2010.

[10] J.M. Borwein, "A note on $\varepsilon$-subgradients and maximal monotonicity", Pacific Journal of Mathematics, vol. 103, pp. 307-314, 1982.

[11] J.M. Borwein and J.D. Vanderwerff, Convex Functions, Cambridge University Press, 2010 .

[12] O. Bueno and B.F. Svaiter, "A non-type (D) operator in $c_{0}$ ", http://arxiv.org/abs/1103.2349v1, March 2011. 
[13] R.S. Burachik and A.N. Iusem, Set-Valued Mappings and Enlargements of Monotone Operators, Springer-Verlag, 2008.

[14] R. Cross, Multivalued Linear Operators, Marcel Dekker, 1998.

[15] M. Fabian, P. Habala, P. Hájek, V. Montesinos Santalucía, J. Pelant and V. Zizler, Functional Analysis and Infinite-Dimensional Geometry, CMS/Springer-Verlag, 2001.

[16] M. Fabian, P. Habala, P. Hájek, V. Montesinos and V. Zizler, Banach Space Theory, CMS/ Springer-Verlag, 2010.

[17] S. Fitzpatrick, "Representing monotone operators by convex functions", in Workshop/Miniconference on Functional Analysis and Optimization (Canberra 1988), Proceedings of the Centre for Mathematical Analysis, Australian National University, vol. 20, Canberra, Australia, pp. 59-65, 1988.

[18] S. Fitzpatrick and R.R. Phelps, "Bounded approximants to monotone operators on Banach spaces", Annales de l'Institut Henri Poincaré. Analyse Non Linéaire, vol. 9, pp. 573-595, 1992.

[19] J.-P. Gossez, "Opérateurs monotones non linéaires dans les espaces de Banach non réflexifs", Journal of Mathematical Analysis and Applications, vol. 34, pp. 371-395, 1971.

[20] J.-P. Gossez, "On the range of a coercive maximal monotone operator in a nonreflexive Banach space", Proceedings of the American Mathematical Society, vol. 35, pp. 88-92, 1972.

[21] W. T. Gowers, "A Banach space not containing $c_{0}, l_{1}$ or a reflexive subspace", Transactions of the American Mathematical Society, vol. .344, pp. 407-420, 1994.

[22] M. Marques Alves and B.F. Svaiter, "A new proof for maximal monotonicity of subdifferential operators", Journal of Convex Analysis, vol. 15, pp. 345-348, 2008.

[23] M. Marques Alves and B.F. Svaiter, "Brøndsted-Rockafellar property and maximality of monotone operators representable by convex functions in non-reflexive Banach spaces", Journal of Convex Analysis, vol. 15, pp. 693-706, 2008.

[24] M. Marques Alves and B.F. Svaiter, "Maximal monotone operators with a unique extension to the bidual", Journal of Convex Analysis, vol. 16, pp. 409-421, 2009

[25] M. Marques Alves and B.F. Svaiter, "On Gossez type (D) maximal monotone operators", Journal of Convex Analysis, vol. 17, pp. 1077-1088, 2010.

[26] R.E. Megginson, An Introduction to Banach Space Theory, Springer-Verlag, 1998. 
[27] R.R. Phelps, Convex Functions, Monotone Operators and Differentiability, 2nd Edition, Springer-Verlag, 1993.

[28] R.R. Phelps, "Lectures on maximal monotone operators", Extracta Mathematicae, vol. 12, pp. 193-230, 1997;

http://arxiv.org/abs/math/9302209v1, February 1993.

[29] R.R. Phelps and S. Simons, "Unbounded linear monotone operators on nonreflexive Banach spaces", Journal of Convex Analysis, vol. 5, pp. 303-328, 1998.

[30] R.T. Rockafellar, "On the maximal monotonicity of subdifferential mappings", Pacific Journal of Mathematics, vol. 33, pp. 209-216, 1970.

[31] R.T. Rockafellar and R.J-B Wets, Variational Analysis, 3rd Printing, Springer-Verlag, 2009.

[32] S. Simons, "The range of a monotone operator", Journal of Mathematical Analysis and Applications, vol. 199, pp. 176-201, 1996.

[33] S. Simons, Minimax and Monotonicity, Springer-Verlag, 1998.

[34] S. Simons, "Five kinds of maximal monotonicity", Set-Valued and Variational Analysis, vol. 9, pp. 391-409, 2001.

[35] S. Simons, From Hahn-Banach to Monotonicity, Springer-Verlag, 2008.

[36] S. Simons, "Banach SSD Spaces and classes of monotone sets", Journal of Convex Analysis, vol. 18, pp. 227-258, 2011.

[37] S. Simons, "Maximal monotone multifunctions of Brøndsted-Rockafellar type", SetValued Analysis, vol. 7 pp. 255-294, 1999.

[38] S. Simons and C. Zălinescu, "Fenchel duality, Fitzpatrick functions and maximal monotonicity", Journal of Nonlinear and Convex Analysis, vol. 6, pp. 1-22, 2005.

[39] M.D. Voisei and C. Zălinescu, "Linear monotone subspaces of locally convex spaces", Set-Valued and Variational Analysis, vol. 18, pp. 29-55, 2010.

[40] L. Yao, "The sum of a maximal monotone operator of type (FPV) and a maximal monotone operator with full domain is maximally monotone", to appear Nonlinear Analysis.

[41] L. Yao, "The sum of a maximally monotone linear relation and the subdifferential of a proper lower semicontinuous convex function is maximally monotone", to appear SetValued and Variational Analysis. 
[42] C. Zălinescu, Convex Analysis in General Vector Spaces, World Scientific Publishing, 2002. 